\title{
SVILUPPO ORGANIZZATIVO DEI NUOVI MOVIMENTI SOCIALI E CONTESTO POLITICO
}

\author{
di Hanspeter Kriesi
}

Nello studio dei movimenti sociali prevalgono ormai due orientamenti: uno incentrato sulla mobilitazione delle risorse e uno incentrato sui processi politici. Il primo approccio si occupa dell'infrastruttura organizzativa dei movimenti sociali, la quale viene considerata il fattore più influente nella loro mobilitazione. Il secondo approccio ha una prospettiva più ampia e situa la mobilitazione dei movimenti sociali all'interno di un contesto politico: la mobilitazione dipende in grande misura dalla struttura delle opportunità politiche che in un dato contesto sono offerte ai movimenti. Sebbene l'approccio basato sui processi politici si sia sviluppato a partire da quello relativo alla mobilitazione delle risorse, rimane carente lo studio dello sviluppo dell'infrastruttura organizzativa dei movimenti sociali in rapporto al contesto politico. La maggior parte degli studi riguardanti i movimenti sociali si è occupata soprattutto delle loro origini, poco del loro sviluppo successivo (McAdam et al. 1988). Inoltre, nella misura in cui si è studiato lo sviluppo organizzativo dei movimenti sociali, esso è stato raramente messo in relazione alle strutture di opportunità politica.

Lo scopo di questo saggio è esaminare il grado in cui lo sviluppo organizzativo dei cosiddetti «nuovi movimenti sociali» dipende dal relativo contesto politico. Prima di affrontare la concettualizzazione e il controllo empirico di questa relazione, è opportuno illustrare $i$ concetti di infrastruttura organizzativa e di sviluppo organizzativo dei movimenti sociali.

Desidero ringraziare Charles Tilly, Marco Giugni e John D. McCarthy per le loro dettagliate osservazioni a una precedente versione di questo saggio, già presentato alla Conferenza sui movimenti sociali tenuta al Life Cycle Institute della Catholic University of America nell'estate 1992. La versione finale inglese sara pubblicata in Opportunities, Mobilizing Structures, and Framing: Comparative Applications of Contemporary Movement Theory, a cura di D. McAdam, J. McCartby e M. Zald.

RIVISTA ITALIANA DI SCIENZA POLITICA / a. XXIII, n. 1, aprile 1993 


\section{Lo sviluppo organizzativo dei movimenti sociali}

Le organizzazioni di movimento sociale sono i mattoni fondamentali dell'infrastruttura organizzativa di un movimento sociale, ma non costituiscono la sua unica componente. Tra le altre componenti vi sono le reti informali di attivisti, le «organizzazioni di supporto» che contribuiscono all'organizzazione sociale degli aderenti a un dato movimento e le «associazioni di movimento» che forniscono alcuni servizi a singoli membri del movimento senza contribuire direttamente alla loro mobilitazione politica.

Un'organizzazione di supporto comprende le organizzazioni di servizio quali i mezzi di informazione, gli esercizi pubblici, le tipografie e le istituzioni educative che intrattengono rapporti amichevoli con il movimento e contribuiscono ai suoi sforzi, senza tuttavia essere completamente integrate nella sua infrastruttura organizzativa ${ }^{1}$. Un'associazione di movimento è un'organizzazione creata appositamente dal movimento al fine di soddisfare alcuni bisogni quotidiani dei propri aderenti ${ }^{2}$ ne sono esempi le organizzazioni di self-belp o di servizio quali le associazioni di mutua assistenza nell'ambito del movimento laburista ${ }^{3}$.

A differenza di queste componenti dell'infrastruttura organizzativa di un movimento, le organizzazioni di movimento sociale sono organizzazioni più $o$ meno formali che si occupano attivamente della mobilitazione politica dei propri aderenti, $i$ quali uniscono le loro forze al fine di sfidare le autorità. In altre parole, un'organizzazione di movimento sociale si distingue dalle altre organizzazioni formali per due motivi: a) essa mobilita i propri membri per azioni collettive, e b) lo fa al fine di ottenere dalle autorità qualche bene collettivo (o la rimozione di qualche «male» collettivo).

Anche le «associazioni di movimento», i gruppi di self-help,

1 Morris qualifica questo tipo di organizzazione come «un gruppo o organizzazione consolidato che è solo parzialmente integrato nella società più ampia, in quanto chi vi partecipa si impegna attivamente in tentativi di provocare cambiamenti nella società» $(1984,139)$.

2 Per Bader le associazioni di movimento fanno parte delle organizzazioni di movimento sociale $(1992,219)$.

${ }^{3}$ Roth (1991) fornisce molti esempi di organizzazioni che contribuiscono all'organizzazione sociale degli ambienti alternativi, nei quali risiede il potenziale politico per $i$ nuovi movimenti sociali in Germania. 
le associazioni di volontariato e $\mathrm{i}$ circoli mobilitano i propri membri, ma nel farlo essi si rivolgono direttamente ai membri e ai clienti. Esse contribuiscono a ciò che Tilly (1978) chiama il catnet delle potenzialità politiche dei movimenti sociali ${ }^{4}, \mathrm{ma}$ non procedono alla mobilitazione per azioni collettive aventi fini politicis. Le «organizzazioni di supporto» lavorano per conto del movimento, il loro personale forse simpatizza con il movimento e partecipa persino alle sue attività; ma non è necessario che sia così, né la partecipazione alle attività del movimento costituisce l'obiettivo principale di queste organizzazioni. Le organizzazioni di movimento sociale, infine, vanno distinte dai partiti e dai gruppi di interesse. A differenza delle prime, in questi ultimi di solito non si ricorre alla partecipazione diretta degli associati al fine di conseguire i propri obiettivi. I partiti e i gruppi di interesse sono specializzati nella rappresentanza politica. Essi dispongono di risorse sufficienti - quali i canali istituzionalizzati di accesso, l'autorità e le conoscenze - per non dover ricorrere alla mobilitazione dei propri membri. Sebbene un partito o un gruppo di interesse possa mobilitare periodicamente i propri membri, ciò non è fondamentale per le loro attività, le quali solitamente vengono svolte da un'élite scelta. Inoltre, questa eventuale mobilitazione tende a manifestarsi secondo procedure di routine ben consolidate. Nella fig. 1 le distinzioni appena fatte vengono riassunte in modo schematico ${ }^{6}$.

L'insieme delle organizzazioni di movimento sociale di un determinato movimento costituisce la sua infrastruttura organizzativa. L'insieme delle infrastrutture organizzative dei movimenti sociali in un determinato sistema politico costituisce, a sua volta, il settore dei movimenti sociali. Le infrastrutture organizzative dei nuovi movimenti sociali rappresentano un sottosetto-

4 Tilly prende in prestito questo termine da Harrison White. Il catnet è un insieme di persone che compongono sia una categoria sia una rete. " $"$ Catnet" riassume in modo elegante il senso di "essere gruppo" che sfugge in locuzioni più complicate» (Tilly 1978, 63).

' La distinzione tra gruppi di self-help, circoli e associazioni di volontariato si riferisce a sua volta alla distinzione tra self-help e altruismo: se i membri e i beneficiari dell'organizzazione sono gli stessi, allora si può parlare di gruppi di self-help o di circoli; altrimenti si ha a che fare con associazioni di volontariato con fini altruisti.

6 Analogamente a quanto fanno McCarthy e Zald $(1977,1218)$, questa concettualizzazione delle organizzazioni di movimento sociale ne restringe l'accezione alle sole organizzazioni complesse e formali; ma essa è più specifica della loro in quanto esclude quelle organizzazioni che condividono le preferenze di un movimento sociale e cercano di attuarle. 


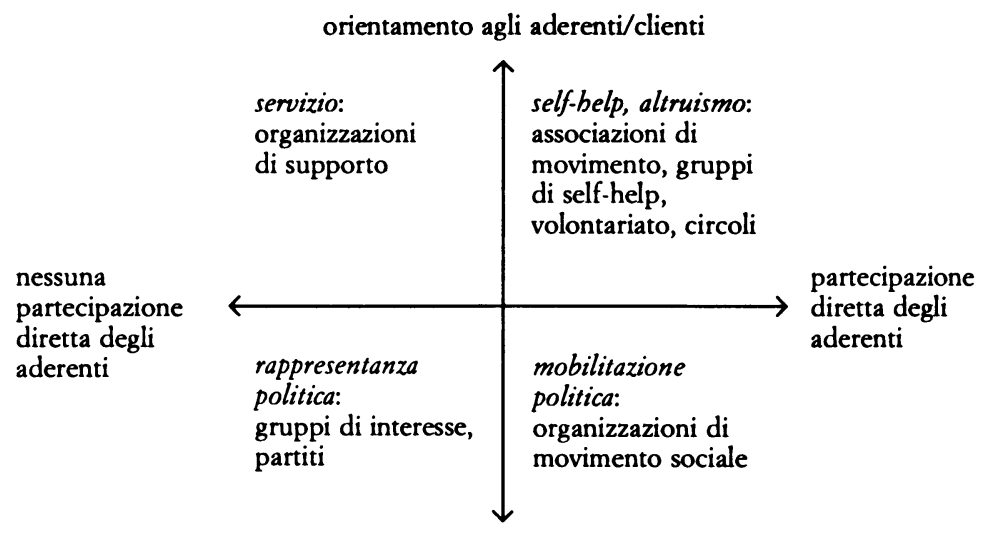

orientamento alle autorità

FIG. 1. Una tipologia delle organizzazioni legate ai movimenti sociali

re del settore dei movimenti sociali ${ }^{7}$. Come osserva Oberschall, un movimento sociale può essere concepito come «un'area di interazione sociale con zone a densità organizzativa variabile» $(1978,267)$. Le organizzazioni di movimento sociale sono soltanto uno degli elementi in questa area di interazione; l'intera rete organizzativa di un movimento sociale si estende ben al di là delle infrastrutture organizzative e comprende altre reti organizzative, di natura sia formale che informale.

\section{I parametri dello sviluppo organizzativo}

Il concetto di sviluppo organizzativo riguarda ogni livello della struttura organizzativa: le organizzazioni di movimento sociale, le infrastrutture organizzative, i sottosettori delle organizzazioni e anche l'intero settore dei movimenti sociali. Esso dunque possiede molte sfaccettature. Propongo quattro insiemi di parametri per analizzarlo: parametri attinenti alla crescita e al declino organizzativi; alla strutturazione organizzativa interna;

7 Seguo ancora le indicazioni di McCarthy e Zald, ma senza adottare l'espressione da loro proposta, «industria dei movimenti sociali», che può essere fuorviante; preferisco pertanto parlare di «infrastrutture organizzative».

${ }^{8}$ Le considerazioni che seguono sono ispirate alle idee sviluppate da Schmitter e Streeck (1981) per l'analisi delle associazioni degli interessi economici. 
alla strutturazione organizzativa esterna; agli obiettivi e ai repertori delle azioni.

La crescita e il declino organizzativi si riferiscono ai cambiamenti delle dimensioni delle infrastrutture organizzative dei movimenti sociali - il numero di organizzazioni di movimento sociale che fanno parte dell'infrastruttura e la quantità di risorse di cui dispongono. Nelle fasi iniziali di un movimento, le uniche risorse che tendono ad essere disponibili sono «l'impegno attivo, il coraggio e la fantasia» degli attivisti e degli aderenti al movimento (Koopmans 1992): le reti organizzative dei movimenti. sociali tendono ad essere deboli e ad avere una struttura informale; gli aderenti di opinione e le élites simpatizzanti stentano ad offrire risorse. I movimenti sono obbligati a richiamare l'attenzione alla loro causa, devono procurarsi da soli i membri e un'élite patrocinante mediante un'esplicita mobilitazione del consenso (Klandermans 1988) o come sottoprodotto della mobilitazione all'azione (McAdam 1982, 125; Jenkins ed Eckert 1986). Di solito, le organizzazioni di movimento sociale si sviluppano esclusivamente nel corso del processo di mobilitazione del movimento sociale. Il flusso di risorse verso la rete organizzativa di un determinato movimento è una funzione dello stato di avanzamento delle campagne di azione volte a crearlo. Un'infrastruttura organizzativa dotata di molte risorse costituisce l'esito, non una causa, della mobilitazione del consenso e delle azioni intraprese da un movimento sociale nascente. A sua volta, il flusso di risorse è un elemento centrale nella determinazione degli altri aspetti dello sviluppo organizzativo.

La strutturazione interna delle organizzazioni di movimento sociale è un prodotto diretto del flusso delle risorse. Questo secondo insieme di parametri si riferisce ai processi di formalizzazione, di professionalizzazione, di differenziazione interna e di integrazione. La formalizzazione corrisponde allo sviluppo di criteri formali di appartenenza, all'introduzione di uno statuto formale e di procedure fisse, alla creazione di una leadership formale e di una struttura ufficiale. La professionalizzazione comporta una gestione per mezzo di funzionari retribuiti, i quali trasformano il loro lavoro per il movimento in una carriera?. La differenziazione interna riguarda la divisione funzionale del

9 McCarthy e Zald (1987) hanno sottolineato come una crescita massiccia nella disponibilità di fondi permette a un numero crescente di professionisti di guadagnarsi un 
lavoro (struttura dei compiti) e il decentramento territoriale (sotto-unità territoriali). L'integrazione delle sotto-unità funzionali e territoriali differenziate si ottiene per mezzo di dispositivi di coordinamento orizzontale e mediante l'accentramento decisionale.

La concentrazione del potere nelle mani di una minoranza dei membri dell'organizzazione - l'oligarchizzazione - è il «dispositivo integrativo» più noto. Mano a mano che le organizzazioni di movimento sociale crescono, cioè all'aumentare delle loro risorse, la loro strutturazione interna si articola progressivamente su ognuna di queste dimensioni. La strutturazione interna è pressoché inevitabile se si vuole che l'organizzazione di movimento sociale abbia successo nel lungo periodo. I verdi tedeschi - i quali non costituiscono un'organizzazione di movimento sociale bensì un partito vicino ai nuovi movimenti sociali - sono un esempio particolarmente cogente dell'inevitabilità di questo processo. I loro difetti strutturali interni hanno vincolato pesantemente le loro possibilità di successo politico (Raschke 1991; Kleinert 1991).

La strutturazione interna favorisce anche la stabilizzazione di un'organizzazione di movimento sociale in periodi di declino organizzativo. McCarthy e Zald sostengono (1977) che le organizzazioni vecchie e consolidate riescono a superare l'intero ciclo di sviluppo e declino organizzativo meglio di quelle sorte di recente. In modo analogo, Staggenborg (1988) asserisce che le organizzazioni formalizzate sono in grado di preservare se stesse e il movimento per periodi di tempo più lunghi rispetto ad organizzazioni informali. Ciò può rivelarsi particolarmente importante in periodi di smobilitazione allorché le issues del movimento sono meno impellenti. Le organizzazioni di movimento sociale formali possono compiere importanti funzioni di mantenimento per il movimento in seguito al successo o alla scomparsa di organizzazioni informali (Jenkins ed Eckert 1986, 827). Infine, come sottolinea ancora Staggenborg, le organizzazioni formali che rimangono in vita in periodi di smobilitazione sono pronte ad approfittarsi di nuove opportunità politiche che potrebbero presentarsi.

Anche la centralizzazione favorisce la stabilità di un'organizzazione di movimento sociale. Taylor (1989) dimostra che, in sociali. 
un periodo in cui il movimento femminista statunitense era in declino, la sua maggiore organizzazione di movimento sociale rimase attiva quasi unicamente a livello nazionale, nell'ambito di una struttura federata in cui le sezioni locali e statali godevano di poca autonomia. La centralizzazione, inoltre, garantisce che gli attivisti del nucleo centrale abbiano un livello relativamente elevato di specializzazione.

La strutturazione esterna si riferisce all'integrazione dell'organizzazione di movimento sociale nel suo ambiente organizzativo. $\mathrm{Vi}$ sono almeno tre dimensioni da tenere presenti a questo proposito: i rapporti dell'organizzazione con i propri membri, con gli alleati e con le autorità. Per definizione un'organizzazione di movimento sociale dipende moltissimo dagli aderen$\mathrm{ti}$, in quanto la sua attività principale consiste proprio nel mobilitarli al fine di intraprendere azioni collettive. Può variare, comunque, il grado di dipendenza dagli aderenti. Un'organizzazione di movimento sociale può ridurre la propria dipendenza da individui il cui livello di impegno è instabile mediante la distribuzione di incentivi selettivi ai propri aderenti: può poi ampliare la propria base di risorse mediante il rafforzamento della propria attrattiva verso potenziali aderenti di opinione, l'offerta di servizi al pubblico o l'ottenimento di sussidi pubblici. La diversificazione della base di risorse in genere fa diminuire la dipendenza da un solo gruppo di appoggio; e ciò è vero non solo per gli aderenti, ma anche per gli alleati e le autorità.

Lo sviluppo delle organizzazioni di movimento sociale avviene in un contesto di interazione assidua con gli alleati e con le autorità. L'appoggio di un alleato potente ha un effetto ambivalente dal punto di vista dello sviluppo dell'organizzazione: da una parte, un alleato potente può essere fonte di risorse; dall'altra, esso può ridurre l'autonomia dell'organizzazione e minacciarne la stabilità di lungo periodo. Allo stesso modo, l'instaurazione di un rapporto di lavoro con le autorità ha effetti ambivalenti sullo sviluppo di un'organizzazione: da una parte, $\mathrm{i}$ riconoscimenti pubblici, l'accesso ai processi decisionali e ai finanziamenti pubblici possono produrre rilevanti flussi di risorse e rappresentare successi importanti; dall'altra, l'integrazione nei sistemi preesistenti di intermediazione degli interessi può porre dei vincoli alla capacità di mobilitazione dell'organizzazione e alienare fasce importanti di aderenti, finendo così per indebolirla nel lungo periodo. 


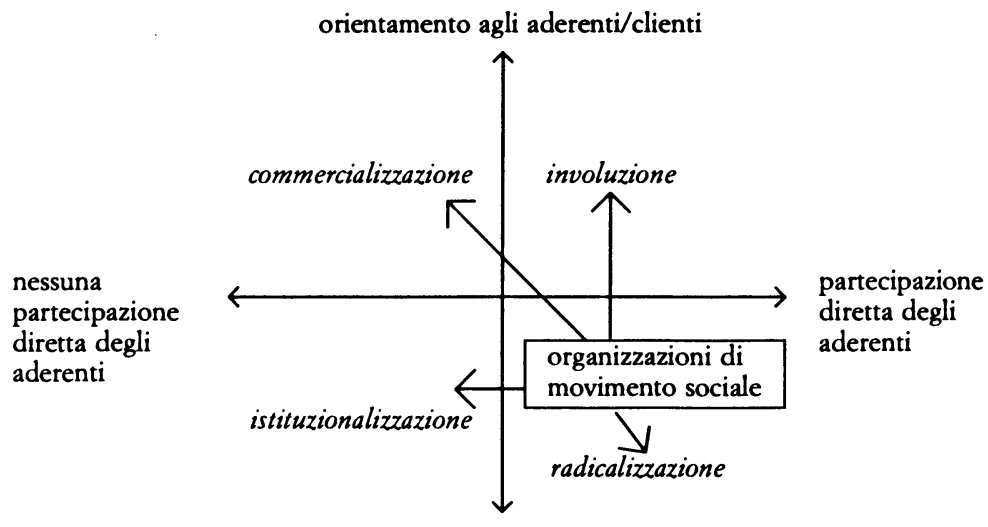

orientamento alle autorità

FIG. 2. Una tipologia delle trasformazioni degli obiettivi e dei repertori delle azioni delle organizzazioni di movimento sociale

Infine, il quarto insieme di parametri di sviluppo organizzativo attiene agli obiettivi e ai repertori di azioni delle organizzazioni di movimento sociale. Secondo il noto modello WeberMichels, le organizzazioni di movimento sociale attraversano tre fasi successive di cambiamento: oligarchizzazione, trasformazione degli obiettivi e slittamento verso il mantenimento organizzativo. In questo modello la trasformazione degli obiettivi si manifesta inevitabilmente in senso conservatore - $\mathrm{i}$ fini dell'organizzazione mutano nella direzione del consenso socialmente dominante. Il mantenimento organizzativo è una forma particolare di trasformazione degli obiettivi in cui l'attività principale dell'organizzazione diventa la conservazione degli aderenti, dei fondi e degli altri presupposti per la sua esistenza. Nel corso di questo processo ci si aspetta che anche il repertorio delle azioni subisca delle modifiche: esso diventerà più moderato, più convenzionale, più istituzionalizzato.

Zald e Ash (1966) criticano questo modello in quanto ritengono che questo tipo di trasformazione, più che essere inevitabile, si manifesta solo in presenza di determinate condizioni. Con uno spirito analogo, vorrei avanzare l'ipotesi che vi siano almeno quattro tipi di trasformazione che possono interessare un'organizzazione di movimento sociale, i quali sono riconducibili ai quattro tipi di organizzazione raffigurati nella fig. 1: un'organizzazione di movimento sociale può avvicinarsi a un 
partito o a un gruppo di interesse; può assumere le caratteristiche di un'organizzazione di supporto o di servizio; può evolversi nella direzione di un gruppo di self-belp, di un'associazione di volontariato o di un circolo; oppure può radicalizzarsi e diventare un'organizzazione ancora più orientata alla mobilitazione per fini di azione collettiva. Nella fig. 2 queste varianti vengono illustrate secondo lo stesso schema della fig. 1.

L'istituzionalizzazione comporta una serie di trasformazioni nel corso delle quali un'organizzazione di movimento sociale si avvicina alla forma-partito o al gruppo di interesse. Queste trasformazioni comportano una stabilizzazione del flusso di risorse, uno sviluppo della struttura interna, una moderazione degli obiettivi, un'evoluzione in senso convenzionale del repertorio delle azioni e l'integrazione nei sistemi di intermediazione degli interessi.

La commercializzazione corrisponde a una trasformazione nella direzione di un'organizzazione di servizio: viene sviluppata l'offerta di servizi a pagamento agli aderenti. Naturalmente, molte organizzazioni ricorrono a «incentivi selettivi» al fine di mobilitare i propri membri per azioni collettive. Secondo la teoria dell'azione collettiva di Olson (1965), di fatto non esistono altri modi per avviare azioni collettive - tranne la coercizione. Tuttavia, l'offerta di incentivi selettivi da parte di un'organizzazione di movimento sociale solitamente non è un fine in sé. Comunque, nella misura in cui diventa un fine in sé, l'organizzazione di movimento sociale si trasforma in un'organizzazione di servizio oppure in un'azienda.

L'involuzione porta a mettere in risalto unicamente gli «incentivi sociali», i quali possono derivare dai servizi di solidarietà forniti agli aderenti oppure dalle attività sociali con altri attivisti dell'organizzazione. Nella prima variante, l'organizzazione di movimento sociale diventa un gruppo di self-help o un'associazione volontaria, a seconda che i beneficiari e gli aderenti coincidano o meno. Nella seconda variante, essa diventa un circolo.

La radicalizzazione, infine, rivitalizza la mobilitazione. Nelle trattazioni classiche dei fenomeni di trasformazione organizzativa, come in quella di Zald e Ash (1966), si parla soprattutto del binomio radicalizzazione-istituzionalizzazione, senza occuparsi delle altre due possibilità. 


\section{I fattori che determinano lo sviluppo organizzativo}

Lo sviluppo organizzativo è una funzione di fattori sia interni che esterni al settore dei movimenti sociali. Per quanto riguarda i fattori interni, si può distinguere la dinamica organizzativa interna dal tipo di movimento. Per quanto riguarda i fattori esterni, vi sono precondizioni generali di tipo economico e culturale e precondizioni politiche. In questa sede, dedicherò maggiore attenzione all'effetto del contesto politico sullo sviluppo organizzativo; ma non ritengo che altri aspetti non siano importanti, né li trascurerò nelle analisi seguenti ${ }^{10}$.

Quanto alla dinamica organizzativa interna, ho già sottolineato che la strutturazione interna può favorire la stabilizzazione nel tempo delle organizzazioni di movimento sociale. Essa potrà anche influire sulle scelte strategiche e tattiche di queste organizzazioni (Staggenborg 1988). Ad esempio, le organizzazioni formalizzate e professionalizzate tendono a ricorrere a tattiche istituzionalizzate e a non intraprendere azioni dirette aventi fini di disturbo. Esse prediligono le tattiche istituzionalizzate in quanto più compatibili con una struttura formalizzata e con gli impegni dei professionisti. Inoltre, la strutturazione interna favorisce l'integrazione nei sistemi esistenti di intermediazione degli interessi: le organizzazioni formalizzate e professionalizzate tendono a godere di un accesso più facile alle autorità pubbliche, in quanto le burocrazie amministrative preferiscono avere a che fare con organizzazioni che hanno procedure operative simili alle loro ${ }^{11}$.

Il tipo di movimento svolge un ruolo centrale nello sviluppo dell'infrastruttura organizzativa. I movimenti si differenziano tra loro specie per quanto attiene ai tipi di aderenti, agli obiettivi perseguiti e al repertorio delle azioni. In questa sede tratterò solo dei cosiddetti «nuovi movimenti sociali», cioè di un insieme di movimenti che sono emersi nell'Europa occidentale a partire dai primi anni settanta. Senza addentrarmi in una disquisizione teorica circa cosa sia un «nuovo movimento socia-

10 Il noto lavoro di Gamson (1975) sulle origini del successo delle organizzazioni di movimento sociale è probabilmente il migliore tentativo di spiegare lo sviluppo organizzativo di queste organizzazioni sulla base di fattori interni. La validità di questo tentativo viene contestata da Goldstone (1980), che evidenzia la rilevanza di fattori politici esterni.

1 Questa interpretazione è propria dell'approccio istituzionale all'analisi organizzativa (Meyer e Rowan 1977; Zucker 1987). 
le», mi limito a stilare un elenco di esempi significativi: il movimento per l'ecologia e il suo ramo anti-nucleare; il movimento per la solidarietà umana e i suoi rami per gli aiuti umanitari, i rifugiati politici, i prigionieri politici, i diritti umani e l'anti-razzismo; il movimento per la pace; il movimento femminista; il movimento di autonomia.

Sebbene si possa sostenere che questi movimenti abbiano un nocciolo di aderenti in comune (Kriesi 1989), in questa sede è opportuno distinguere tra tre tipi di nuovo movimento sociale (Koopmans 1990a): strumentale, subculturale e controculturale. I movimenti strumentali cercano di ottenere determinati beni collettivi o di impedire determinati «mali» collettivi. Non si curano in modo precipuo dell'identità collettiva dei propri aderenti. Il movimento per la pace, il movimento per l'ecologia e quello solidaristico ne sono esempi. Di converso, i movimenti subculturali, come il movimento degli omosessuali e (alcuni settori di) quello femminista, puntano alla (ri-)produzione di un'identità collettiva, costituita in via primaria mediante l'interazione interna ma che dipende anche da azioni dirette verso le autorità. Anche i movimenti controculturali, come quello di autonomia, sono incentrati sull'identità, ma essi la costituiscono soprattutto mediante un'interazione conflittuale con le autorità o con terzi.

Le differenze nell'orientamento generale dei tre tipi di movimento tendono a influenzare il loro sviluppo organizzativo in modi significativi. È probabile che il livello e il tipo di risorse di cui dispone l'infrastruttura organizzativa di un movimento sociale siano una funzione dell'orientamento generale del movimento. Ne consegue che di solito le organizzazioni dei nuovi movimenti sociali di tipo strumentale saranno inclusive, cioè cercheranno di mobilitare quanta più gente possibile al fine $\mathrm{di}$ conseguire il loro obiettivo specifico. Già per questo solo motivo ci si aspetta che ad esse aderiranno più individui e confluiranno più risorse che non a movimenti più orientati all'identità. L'identità si collega a specifiche caratteristiche degli aderenti, il che implica l'esclusività e un numero più circoscritto di membri. D'altra parte, è più probabile che le infrastrutture di movimenti sociali esclusivi, orientati all'identità, riescano a sollecitare un impegno più intenso da parte dei propri membri. In vista della stabilità di lungo termine, ciò costituisce un'alternativa alla strutturazione interna. Nella sua indagine sulle comuni statunitensi, Kanter (1972) conclude che i gruppi caratterizzati da 
un maggiore impegno tendono a trattenere $\mathrm{i}$ loro aderenti e a durare nel tempo. L'impegno è particolarmente importante per tenere in vita un'organizzazione di movimento sociale quando non ci si trova in una fase di mobilitazione di massa (Taylor 1989). Questo ragionamento spinge le infrastrutture dei movimenti sub- e controculturali ad opporre molta resistenza al declino dei propri movimenti.

Per quanto riguarda i movimenti strumentali, l'istituzionalizzazione del flusso di risorse pare essere particolarmente problematica per tutti i movimenti molto incentrati intorno a una singola issue, nonché per i movimenti che si occupano di problemi internazionali. Più un movimento si occupa di un unico problema, più esso dipende dal ciclo di attenzione pubblica per le issues. Specie per quanto riguarda i problemi internazionali, questo ciclo sembra essere piuttosto breve. Inoltre, i problemi internazionali tendono a comportare una forte polarizzazione partitica e politica, il che introduce un ulteriore elemento di incertezza (vedi oltre). Con queste premesse, i movimenti per la pace e per la solidarietà dovrebbero avere maggiori difficoltà a rendere stabili le loro basi di risorse rispetto al movimento per l'ecologia. Tranne che per il suo ramo anti-nuclea$\mathrm{re}$, il quale è piuttosto incentrato intorno a un unico problema, il movimento per l'ecologia deve fare $i$ conti con una struttura di problemi altamente differenziata, complessa e di livello nazionale. Infine, è probabile che il tipo di movimento abbia un effetto sul modello di trasformazione delle infrastrutture organizzative: è probabile che i movimenti strumentali si istituzionalizzino, i movimenti subculturali seguano le vie dell'involuzione o della commercializzazione, i movimenti controculturali si radicalizzino.

Oltre ai fattori interni, anche alcuni fattori esterni al settore dei movimenti sociali ne determinano lo sviluppo. Lo sviluppo economico di un paese influisce sul livello generale di risorse a disposizione per il settore. Seguendo il ragionamento di McCarthy e Zald (1977), se la quantità di risorse in una società aumenta, gli imprenditori politici saranno spinti a fondare nuove organizzazioni di movimento sociale di tipo professionale, le quali competeranno per queste risorse e contribuiranno allo sviluppo organizzativo del movimento. Anche i fattori culturali possono svolgere un ruolo: ad esempio, i diversi livelli di partecipazione ad «associazioni volontarie» in paesi diversi sono stati attribuiti alla presenza o meno di una cultura politica anglo-sas- 
sone e di una forte tradizione protestante (Curtis et al. 1992)12. In questo saggio mi occuperò soprattutto di come i fattori politici influenzino lo sviluppo organizzativo dei nuovi movimenti sociali. Al fine di studiare questi effetti ricorrerò al concetto di struttura di opportunità politica, che ho sviluppato altrove (Kriesi 1991) e che è stato già usato per un'analisi comparata dei livelli di mobilitazione (Kriesi et al. 1992). Per l'esposizione e per il controllo empirico delle mie ipotesi ricorrerò agli stessi quattro paesi usati in quell'analisi: Francia, Germania, Paesi Bassi e Svizzera. È probabile che i fattori politici non abbiano effetti diretti sulla strutturazione interna di un'infrastruttura organizzativa di un movimento sociale. Essi sembrano invece importanti per determinare il livello e il tipo di risorse di cui un'infrastruttura organizzativa può disporre, per la sua strutturazione interna e per i modelli generali di trasformazione che caratterizzeranno il suo sviluppo organizzativo.

\section{Il contesto politico}

La struttura delle opportunità politiche può essere disarticolata in tre grandi insiemi di proprietà: la struttura istituzionale formale di un sistema politico; le procedure informali e le strategie prevalenti del sistema dinanzi alle sfide; e la configurazione del potere per quanto concerne i rapporti con gli sfidanti. I primi due insiemi costituiscono il contesto generale per lo sviluppo organizzativo e delimitano la corrispondente configurazione del potere. La struttura istituzionale formale determina la forza complessiva dello stato. Questa può anche variare nel tempo a causa delle vicende elettorali, di decisioni strategiche o della dinamica delle interazioni tra sfidanti e autorità.

Sulla base dei primi due insiemi, ho elaborato una tipologia dei contesti generali con quattro tipi, ognuno dei quali corrisponde a uno dei quattro paesi summenzionati. La tipologia è illustrata nella fig. 3. La combinazione di uno stato forte e di una strategia dominante di tipo esclusivo dà luogo a una situazione di esclusione selettiva degli sfidanti. In una situazione come questa, lo sfidante non può contare su un accesso né for-

12 L'etichetta «associazioni volontarie» è stata usata anche per le organizzazioni di movimento sociale e per i gruppi di interesse. 


\begin{tabular}{|c|c|c|}
\hline \multirow{2}{*}{$\begin{array}{l}\text { Strategia } \\
\text { dominante }\end{array}$} & \multicolumn{2}{|c|}{ Struttura istituzionale formale } \\
\hline & Stato debole & Stato forte \\
\hline \multirow[t]{3}{*}{ Esclusiva } & inclusione formalista & esclusione selettiva \\
\hline & $\begin{array}{l}\text { - facilitazione formale, } \\
\text { ma non informale, } \\
\text { all'accesso } \\
\text { - forte repressione } \\
\text { - possibilità di veto, } \\
\text { ma nessuna concessione } \\
\text { sostanziale }\end{array}$ & $\begin{array}{l}\text { - nessuna facilitazione } \\
\text { all'accesso, né formale } \\
\text { né informale } \\
\text { - forte repressione } \\
\text { - nessuna possibilità di } \\
\text { veto, né concessioni } \\
\text { sostanziali, a seconda } \\
\text { della configurazione } \\
\text { del potere }\end{array}$ \\
\hline & (Germania) & (Francia) \\
\hline \multirow[t]{3}{*}{ Inclusiva } & $\begin{array}{l}\text { integrazione } \\
\text { procedurale totale }\end{array}$ & cooptazione informale \\
\hline & $\begin{array}{l}\text { - facilitazione sia } \\
\text { formale sia informale } \\
\text { all'accesso } \\
\text { - } \text { repressione debole } \\
\text { - possibilità di veto, ma } \\
\text { nessuna concessione }\end{array}$ & $\begin{array}{l}\text { - facilitazione informale, } \\
\text { ma non formale, } \\
\text { all'accesso } \\
\text { - repressione debole } \\
\text { - concessioni sostanziali, } \\
\text { ma nessuna possibilità } \\
\text { di veto }\end{array}$ \\
\hline & (Svizzera) & (Paesi Bassi) \\
\hline
\end{tabular}

FIG. 3. Una tipologia delle reazioni dei governi agli sfidanti

male né informale al sistema politico. Lo stato, in virtù della sua forza, può anche scegliere di ignorare gli sfidanti; ma se reagisce, probabilmente lo farà per reprimere lo sfidante. Inoltre, dato che lo stato è forte, lo sfidante probabilmente non disporrà di alcuna capacità di veto; ma, a seconda della composizione del governo, potrà forse ottenere qualche concessione sostanziale. Ad esempio, un governo di sinistra potrebbe far sì che lo stato faccia delle concessioni a favore dei nuovi movimenti sociali che gli lanciano delle sfide $^{13}$. La Francia è un esempio di questo tipo di contesto generale.

\section{All'estremo opposto dell'esclusione selettiva si trova il caso}

${ }^{13}$ Nella prima concettualizzazione di questo tipo non avevo tenuto conto di questa possibilità di concessioni selettive, la quale è stata indicata da Duyvendak (1992). Sulla base del suo suggerimento, ho cambiato il nome di questo tipo da «esclusione totale» ad «esclusione selettiva». 
dell'integrazione procedurale totale, caratterizzato dalla compresenza di uno stato debole e di una strategia dominante di tipo inclusivo. In una situazione come questa, la repressione è debole e l'accesso al sistema da parte dello sfidante viene facilitato sia formalmente che informalmente. Stante la debolezza del sistema, lo sfidante non può contare su concessioni sostanziali di rilievo, ma potrà bloccare alcune decisioni mediante l'esercizio di un veto. La Svizzera rappresenta un esempio di questo tipo: le sue istituzioni di democrazia diretta e la struttura federale fanno sì che gli sfidanti dispongano di molti canali di accesso formale. Inoltre, la strategia tradizionalmente integrativa rinforza l'effetto generale della struttura formale.

La Germania rappresenta uno dei due tipi intermedi, quello dell'inclusione formalista. In questo caso, lo sfidante può contare su un accesso formale facilitato, ma non su canali informali. Vi è la possibilità di veto, ma non ci si deve attendere delle concessioni. La struttura federale della Germania permette una molteplicità di punti di accesso; inoltre, il sistema giudiziario offre agli sfidanti un'ulteriore serie di punti di accesso. La tradizione repressiva del sistema, d'altra parte, fa sì che chi si esprime al di fuori dei canali formali vada incontro a una forte repressione.

Il secondo tipo intermedio, quello della cooptazione informale, corrisponde al caso olandese. In questo caso, gli sfidanti non godono di molti punti di accesso formale, ma risulta agevole l'accesso informale. Dato che lo stato olandese è anche piuttosto forte, esso può fare delle notevoli concessioni sostanziali e impedire agli sfidanti di esercitare un veto.

Come aveva già osservato Tocqueville, uno stato forte si accompagna a una società debole e viceversa. La mancanza di un accesso decentrato allo stato scoraggia l'auto-organizzazione ai livelli locali e di base. Anche una strategia dominante di tipo repressivo serve a scoraggiare l'auto-organizzazione. Fedele alla tradizione della Rivoluzione francese, lo stato francese osteggia da molto tempo la formazione di associazioni di qualsiasi tipo. Perciò, lo sviluppo organizzativo della maggior parte delle associazioni francesi è per tradizione meno robusto che in altri paesi. Ne sono indizi la relativa debolezza dei sindacati francesi (Rosanvallon 1988) e delle associazioni di volontariato in genere (Curtis et al. 1992). Tilly et al. $(1975,43)$ hanno sostenuto che questa debolezza delle organizzazioni francesi è più apparente che reale: le associazioni francesi spesso nascono da organizza- 
zioni preesistenti - come la chiesa cattolica, il partito comunista e così via - e, benché siano attive, molte di esse non giungono mai ad avere una formale esistenza giuridica. L'attiva opposizione dello stato ad associazioni formali, inoltre, può aver rafforzato la tendenza a formare questi gruppi senza troppo rumore. Tuttavia, le strutture informali e la dipendenza da appoggi esterni non costituiscono segni di sviluppo organizzativo. Infatti, le argomentazioni dei Tilly mettono in luce un ulteriore meccanismo che contribuisce a indebolire la struttura organizzativa francese (vedi oltre).

Di converso, al debole stato svizzero corrisponde una società altamente organizzata. L'auto-organizzazione della società svizzera è molto avanzata, e lo stato centrale interviene solo se è necessario. In base al principio di sussidiarietà, infatti, i problemi sociali vengono affrontati in prima istanza da organizzazioni private, poi dai livelli locali o regionali dell'amministrazione pubblica. Tocca allo stato centrale trovare una soluzione solo qualora gli attori precedentemente menzionati non vi riescano.

A prima vista, il forte stato centralizzato dei Paesi Bassi è meno propizio allo sviluppo organizzativo che non il debole stato federale svizzero. Tuttavia, per quanto riguarda la stimolazione dell'auto-organizzazione della società, la pillarization olandese rappresenta da tempo un equivalente funzionale del federalismo svizzero (Kriesi 1990). La pillarization comporta la costruzione di infrastrutture organizzative parallele in diversi ambiti della vita sociale. Se federalismo e pillarization hanno entrambi stimolato l'auto-organizzazione, i loro effetti sulla strutturazione organizzativa sono molto diversi: il federalismo è una struttura statale che fa sì che, per quanto possibile, non si intervenga in alcun modo nello sviluppo delle organizzazioni sociali; di converso, la pillarization è una forma di struttura sociale che è sempre esistita accanto a un forte stato centralizzato, il quale a sua volta ha sempre facilitato l'auto-organizzazione mediante una strategia di cooptazione informale. Queste facilitazioni si estrinsecano nel riconoscimento pubblico, nella partecipazione in forma consultiva ai processi decisionali, persino nella concessione di sussidi.

$\mathrm{Ci}$ aspetteremmo, pertanto, che le infrastrutture organizzative dei movimenti sociali in Olanda fossero sviluppate almeno tanto quanto quelle svizzere, forse anche di più. Secondo lo studio comparato di Curtis et al. (1992), nei Paesi Bassi, effettivamente, si registrano dei livelli molto elevati di adesione alle 
associazioni volontarie: se si escludono le appartenenze a chiese e a sindacati, il livello olandese è il più alto tra quelli dei paesi presi in esame ${ }^{14}$. La Svizzera non era inclusa in questa indagine, ma si possono trarre alcune informazioni utili da una ricerca sui valori degli svizzeri (Melich 1992): in Svizzera, il livello di adesione ad associazioni volontarie è esattamente pari a quello olandese, purché si escludano le appartenenze a chiese e a sindacati (entrambe sono relativamente marginali in questo paese) ${ }^{15}$.

La Germania, infine, rappresenta un caso intermedio. Il grado di apertura del debole stato tedesco stimola l'auto-organizzazione della società, ma la tradizione repressiva e la distanza che in questo paese tradizionalmente separa stato e società suggeriscono che, rispetto alla sfera pubblica, questa auto-organizzazione sia meno sviluppata che non nei due paesi più piccoli. Sontheimer $(1989,111)$ sostiene che la Germania è caratterizzata da una sfera privata ben organizzata, la quale, però, non è sufficientemente integrata nella struttura della sfera pubblica. In questo paese, la sfera pubblica e quella privata non sarebbero automaticamente complementari. Almond e Verba (1963), ad esempio, avevano trovato un livello di adesione ad associazioni volontarie molto elevato in Germania; ma l'incidenza degli attivisti era bassa come in Italia o in Messico. I due studiosi interpretarono questo risultato come segue: benché le strutture politiche tedesche fossero ben sviluppate, esse non svolgevano (ancora) un ruolo di rilievo nella vita dei cittadini. Rispetto ai primi anni sessanta, l'atteggiamento dei cittadini nei confronti del loro stato è migliorato, e la partecipazione politica è aumentata in modo significativo. La Germania è diventata una «normale» democrazia occidentale (Rudzio 1987; Koopmans 1992). Secondo i dati di Curtis et al. (1992), il tasso di adesione ad associazioni volontarie in Germania, se si escludono le chiese e i sindacati, è leggermente superiore alla media di tutti i paesi presi in esame; ed è senz'altro più elevato che in Francia, ma più basso che nei Paesi Bassi o in Svizzera.

14 I 15 paesi compresi nell'indagine di Curtis et al. (1992) sono, oltre agli-altri tre paesi esaminati in questo saggio, i paesi anglosassoni (Stati Uniti, Canada, Australia, Irlanda, Irlanda del Nord e Gran Bretagna), Italia, Spagna, Belgio, Norvegia, Svezia e Giappone.

15 Queste informazioni non sono state pubblicate. Sono state elaborate da me, a partire dai dati grezzi. 


\section{La configurazione del potere}

Per quanto riguarda il rapporto tra configurazione di potere nel sistema dei partiti e nuovi movimenti sociali, ho sostenuto altrove (Kriesi 1991) che è opportuno dedicare un'attenzione particolare alla configurazione del potere a sinistra. In generale, chi aderisce ai nuovi movimenti sociali proviene dalla sinistra politica. I partiti di destra hanno tentato solo di recente di rispondere ad alcune esigenze poste dai nuovi movimenti sociali, soprattutto nell'ambito della politica ambientale. Con ogni probabilità, mano a mano che i partiti di destra si aprono alle nuove rivendicazioni, anche la loro configurazione di potere assumerà maggiore importanza per i nuovi movimenti sociali. Sono particolarmente rilevanti due aspetti della configurazione del potere a sinistra: a) la divisione o meno della sinistra in una grande componente comunista e una seconda componente socialdemocratica o socialista, e b) la partecipazione o meno della sinistra al governo.

Solo in Francia, tra i quattro paesi in esame, la sinistra è divisa; ciò ha comportato una forte polarizzazione all'interno della sinistra, oltre a quella tra la sinistra nel suo complesso e la destra. Se la sinistra è divisa, le sue due maggiori componenti cercano di strumentalizzare i nuovi movimenti sociali: innanzitutto per ottenere una posizione egemone nell'ambito della sinistra, in secondo luogo per prevalere nei confronti della destra nelle elezioni. È probabile che una qualsiasi strumentalizzazione politica da parte di un partito abbia un effetto destabilizzante sullo sviluppo organizzativo di un'infrastruttura, in quanto quest'ultima viene a dipendere dalle vicissitudini della politica partitica. Infatti, nella misura in cui cambia la natura della competizione interpartitica, la sinistra potrebbe ritirare il suo appoggio all'infrastruttura.

In Francia accadde proprio questo quando la sinistra andò al potere nel 1981 (Duyvendak 1992). In questo caso, le ripercussioni sulle risorse a disposizione delle infrastrutture organizzative dei nuovi movimenti sociali furono molto gravi, data l'elevata dipendenza di questi ultimi dai partiti di sinistra. Inoltre, la strategia comunista nei confronti dei nuovi movimenti sociali ebbe degli effetti particolarmente negativi per lo sviluppo delle loro infrastrutture. Mentre i socialdemocratici tendono a stringere alleanze poco impegnative con organizzazioni già esistenti nell'ambito di un determinato movimento sociale, i co- 
munisti tendono a istituire organizzazioni proprie al fine di controllare l'infrastruttura del movimento che a loro interessa (Garner Ash e Zald 1987, 313 e ss.). La presenza di organizzazioni colonizzate dal partito comunista introduce inevitabilmente delle tensioni competitive all'interno dell'infrastruttura, in quanto non tutte le organizzazioni vorranno unirsi alla causa comunista e la maggior parte di esse rifiuteranno la leadership pretesa dai comunisti. Va sottolineato che la strategia comunista per il controllo delle infrastrutture organizzative dei movimenti non ha toccato nella stessa misura tutti i nuovi movimenti sociali. Essa è stata particolarmente intensa nel caso dei nuovi movimenti sociali di tipo strumentale, che si occupano di problemi internazionali inerenti al confronto tra regimi ex-comunisti e l'Occidente, ad esempio i movimenti per la pace e per la solidarietà. La strategia comunista ha influito poco, al contrario, sul movimento per l'ecologia. A parità di condizioni, pertanto, ci si aspetta che in Francia il movimento per la pace e quello solidaristico abbiano delle infrastrutture organizzative particolarmente deboli.

La strumentalizzazione politica dei nuovi movimenti sociali da parte dei partiti, con tutte le implicazioni negative che essa comporta per la stabilità delle infrastrutture organizzative dei movimenti, è meno marcata negli altri tre paesi. Ma anche in essi la sinistra ha tentato di strumentalizzare alcuni movimenti: i socialdemocratici olandesi, ad esempio, hanno sostenuto il movimento per la pace nella sua campagna contro i missili cruise. Dopo la sconfitta alle urne nel 1986, accortisi che questo appoggio non portava benefici elettorali, $i$ socialdemocratici abbandonarono il movimento, contribuendo così al suo declino (Van Praag 1992). Si tratta di un esempio insolito di un partito socialdemocratico all'opposizione che ritira il proprio appoggio a un nuovo movimento sociale. Spostandosi verso il centro, $i$ socialdemocratici speravano di tornare ad essere, agli occhi dei democristiani, candidati appetibili per una coalizione governativa. In un altro caso atipico, un governo socialista ha favorito la mobilitazione di un nuovo movimento: i socialisti francesi hanno aiutato Sos Racisme, rivitalizzando così il movimento solidaristico francese. L'appoggio dei socialisti a questo movimento faceva parte della loro strategia contro l'estrema destra, la quale si faceva sempre più minacciosa in termini elettorali (Duyvendak 1992). Questi due esempi evidenziano il carattere relativo della regola generale secondo la quale un partito di sinistra al 
potere non favorirà la mobilitazione di nuovi movimenti sociali, e un partito di sinistra all'opposizione, invece, li appoggerà.

\section{Un controllo empirico}

Nel quadro di un progetto di ricerca sullo sviluppo dei nuovi movimenti sociali in Europa occidentale nel corso degli anni ottanta, sono state raccolte in modo sistematico alcune informazioni sullo sviluppo organizzativo di cinque nuovi movimenti sociali nei quattro paesi summenzionati ${ }^{16}$. Tra i movimenti per i quali sono state raccolte le informazioni sono compresi i tre movimenti di tipo strumentale di maggiore rilievo (i movimenti per la pace, per l'ecologia e per la solidarietà), un movimento subculturale (il movimento degli omosessuali) e un movimento controculturale (il movimento di autonomia). In un primo tempo intendevamo studiare quantomeno le cinque organizzazioni più importanti nell'ambito di ciascun movimento; ma per una serie di motivi il numero di organizzazioni effettivamente esaminate è risultato più variabile del previsto. In alcuni casi non disponevamo di informazioni su cinque organizzazioni, soprattutto per problemi di accesso (come nel caso del movimento di autonomia) ma anche perché a volte non esistevano cinque organizzazioni di un certo rilievo (come nel caso del movimento degli omosessuali in Germania e in Svizzera). Per alcuni movimenti di tipo strumentale sono state prese in considerazione più di cinque organizzazioni, per lo più per esigenze interne alle ricerche nazionali. La natura non uniforme della base dei dati pone un problema di rappresentatività. Di conseguenza, a volte prenderò in esame sottoinsiemi di organizzazioni invece del campione intero. La tab. 1 riporta in modo sintetico le caratteristiche delle organizzazioni che compongono il campione.

La rilevazione dei dati è stata effettuata mediante la raccolta di documenti e l'effettuazione di interviste personali altamente strutturate a rappresentanti delle organizzazioni di movimento sociale. Le informazioni riguardano tutti gli aspetti dello svilup-

${ }^{16}$ La raccolta delle informazioni è stata effettuata da Jan Willem Duyvendak in Francia; Ruud Koopmans in Germania; Florence Passy e Marco Giugni in Svizzera; Luc Wijmans, Hein-Anton van der Heijden e un gruppo di studenti coordinati da Jan Willem Duyvendak e Ruud Koopmans nei Paesi Passi. 
ТАВ. 1. Distribuzione delle organizzazioni del campione per paese e per movimento

\begin{tabular}{lccccc}
\hline & \multicolumn{5}{c}{ Paesi } \\
Movimenti & Paesi Bassi & Germania & Francia & Svizzera & Totale \\
\hline Pace & 2 & 4 & 5 & 6 & 17 \\
Ecologia & 10 & 11 & 4 & 10 & 35 \\
Solidarietà umana & 7 & 8 & 8 & 13 & 36 \\
Omosessuali & 4 & 3 & 7 & 3 & 17 \\
Autonomia & 1 & 2 & 1 & 5 & 9 \\
Totale & 24 & 28 & 25 & 37 & 114 \\
\hline
\end{tabular}

po organizzativo menzionati in precedenza, riferiti in genere al periodo 1988-89. Per alcuni aspetti abbiamo richiesto anche informazioni approssimative relative alle tendenze degli anni ottanta. In qualche caso siamo riusciti ad avere dati relativi alla consistenza dell'adesione e alle risorse finanziarie su base annua per il periodo 1975-1989. La qualità dei dati, purtroppo, è piuttosto scadente: ci sono molti dati mancanti e molte stime approssimative ottenute mediante valutazioni ragionate degli intervistati o per mezzo di un'interpolazione da parte mia. Le organizzazioni di movimento sociale di solito non dedicano molti sforzi alla tenuta di archivi dettagliati sul proprio sviluppo. Anche laddove questi archivi esistono, non sempre gli attuali addetti hanno potuto o voluto recuperarli e renderli accessibili per i nostri scopi euristici. Dato il limitato numero dei casi e la bassa qualità della rilevazione, l'analisi che segue sarà piuttosto semplice.

Oltre a queste informazioni, ricorrerò a dati esaurienti relativi ad eventi di protesta nel periodo 1976-1988 nei quattro paesi in questione. In ogni paese abbiamo esaminato l'edizione del lunedì di un quotidiano importante per l'intero periodo di cui sopra. Per ogni evento, sono state codificate alcune informazioni, tra cui le organizzazioni promotrici (fino a tre in numero). Ciò consente di studiare lo sviluppo del repertorio delle azioni dei vari tipi di organizzazione nei quattro paesi.

Il resto del saggio consiste nella presentazione di alcuni risultati inerenti a ciascuno dei quattro insiemi di parametri relativi allo sviluppo organizzativo. 


\section{Sviluppo e declino organizzativo}

Il numero dei membri e l'ammontare delle risorse finanziarie sono i due indicatori del livello di risorse a disposizione. Le organizzazioni più importanti di un movimento sociale sono, in generale, quelle più dotate di risorse; si è comunque fatto uso anche di altri criteri per determinare quali fossero le organizzazioni più importanti. Alcune organizzazioni sono importanti in virtù della loro capacità di mobilitazione, anche se non dispongono di molti aderenti o di molte risorse finanziarie. La Bbu tedesca (Lega federale delle iniziative civiche per la tutela ambientale), ad esempio, è un'associazione di associazioni (peak association) del movimento per l'ecologia che non ha membri diretti e dispone solo di poche risorse; ma nel periodo preso in esame essa possedeva una capacità di mobilitazione impressionante ed esercitava molta influenza sull'opinione pubblica (Rucht 1989, 73).

Farò ricorso a tre tipi di confronti tra i livelli di risorse: a) una comparazione di quattro organizzazioni internazionali con sezioni nei quattro paesi in esame; $b$ ) una comparazione delle quattro organizzazioni più grandi in ciascuno dei quattro paesi; c) una comparazione di tutte le organizzazioni del campione a seconda del paese e del movimento. I quattro paesi, evidentemente, hanno dimensioni molte diverse: la Germania e la Francia sono circa quattro volte più popolose dei Paesi Bassi e dieci volte più popolose della Svizzera. Al fine di tener conto di queste differenze, nelle tabelle vengono riportati sia i valori assoluti sia i valori relativi, sotto forma di incidenze per ogni milione di abitanti.

TAB. 2. Numero di aderenti (in migliaia) a quattro organizzazioni di movimento sociale nel 1989 in quattro paesi

Paesi

\begin{tabular}{lcccc} 
Organizzazioni & Paesi Bassi & Germania & Francia & Svizzera \\
\hline Greenpeace & 640 & 600 & 5 & 24 \\
Wwf & 100 & 75 & $-a$ & 130 \\
Amnesty International & 120 & 23 & 21 & 47 \\
Terre des Hommes & 66 & 40 & 28 & 30 \\
Totale aderenti & 926 & 738 & 54 & 231 \\
$\begin{array}{l}\text { N. aderenti ogni } \\
\text { mille abitanti }\end{array}$ & 62 & 12 & 1 & 36 \\
\hline
\end{tabular}

a L'informazione non è disponibile. 
La tab. 2 riporta alcuni confronti relativi al numero di aderenti a quattro organizzazioni: Greenpeace e il Fondo mondiale per la natura (Wwf) per il movimento per l'ecologia; Amnesty International e Terre des Hommes per il movimento solidaristico. I risultati sono univoci: in termini sia relativi che assoluti, queste organizzazioni sono forti innanzitutto nei Paesi Bassi, poi in Germania (in termini assoluti) e in Svizzera (in termini relativi); le loro sezioni francesi sono invece molto deboli. Spiccano le dimensioni eccezionali delle sezioni olandese e tedesca di Greenpeace, il cui peso influenzerà le analisi che seguono.

Se per ogni paese si confrontano le quattro organizzazioni più grandi nell'ambito di ciascun movimento, i risultati appena presentati vengono per lo più confermati ${ }^{17}$. Di nuovo, le organizzazioni francesi sono di gran lunga le più deboli. Le organizzazioni svizzere ora sembrano forti quanto quelle olandesi, purché si tenga conto della diversa popolosità dei due paesi. In termini assoluti, nelle organizzazioni olandesi vi è un numero più elevato di membri nonostante il paese sia più piccolo della Germania e della Francia. In termini relativi, le organizzazioni tedesche sono situate in una posizione intermedia.

Nella tab. 3 si riscontrano anche differenze sistematiche nei livelli di adesione ai diversi movimenti, differenze che prescindono dal paese. In linea con le nostre aspettative, i movimenti strumentali raccolgono un maggior numero di aderenti rispetto a quelli subculturali ${ }^{18}$. Inoltre, tra $\mathrm{i}$ movimenti strumentali, quello per l'ecologia è di gran lunga il più grande, e quello per la pace il più piccolo. L'unica eccezione è la Francia, dove le quattro maggiori organizzazioni del movimento solidaristico hanno più iscritti che non le loro controparti nel movimento per l'ecologia. Ciò è dovuto al fatto che, come si è già accennato, il governo socialista ha appoggiato in modo consistente il movimento per la solidarietà e non ha appoggiato affatto quello per l'ecologia. Anzi, il governo socialista ha addirittura aggredi-

${ }^{17}$ Sono state considerate soltanto organizzazioni che prevedono adesioni personali. Non si è tenuto conto, in altri termini, di peak associations, ovvero di organizzazioni di organizzazioni. Così, ad esempio, non è stato considerata la Federazione francese delle associazioni per la tutela della natura (Ffspn), alla quale fanno capo 850.000 persone, né l'Associazione tedesca per la tutela della natura (Dnr), che raggruppa circa 100 associazioni per un totale di 3,3 milioni di persone.

${ }_{18}$ Il movimento di autonomia non è stato incluso nella tab. 3 in quanto le informazioni raccolte sono insufficienti. 
TAв. 3. Numero di aderenti nel 1989 alle quattro organizzazioni più importanti di ogni movimento sociale in quattro paesi

\begin{tabular}{|c|c|c|c|c|}
\hline \multirow{2}{*}{$\begin{array}{l}\text { Movimento di } \\
\text { riferimento per le } \\
\text { organizzazioni }\end{array}$} & \multicolumn{4}{|c|}{ Paesi } \\
\hline & Paesi Bassi & Germania & Francia & Svizzera \\
\hline \multicolumn{5}{|l|}{$\begin{array}{l}\text { Valori assoluti } \\
\text { (in migliaia) }\end{array}$} \\
\hline $\begin{array}{l}\text { Ecologia } \\
\text { Solidarietà } \\
\text { Pace } \\
\text { Omosessuali }\end{array}$ & $\begin{array}{r}1026 \\
165^{a} \\
-b \\
24^{a}\end{array}$ & $\begin{array}{r}1024 \\
113 \\
25^{a} \\
19^{a}\end{array}$ & $\begin{array}{l}12^{4} \\
77 \\
31 \\
35\end{array}$ & $\begin{array}{r}373 \\
93 \\
16 \\
14\end{array}$ \\
\hline $\begin{array}{l}\text { Totale aderenti } \\
\text { N. aderenti per ogni } \\
\text { milione di abitanti }\end{array}$ & $\begin{array}{r}1215 \\
81\end{array}$ & $\begin{array}{r}1181 \\
19\end{array}$ & $\begin{array}{r}155 \\
3\end{array}$ & $\begin{array}{r}483 \\
74\end{array}$ \\
\hline \multicolumn{5}{|l|}{ Valori percentuali } \\
\hline $\begin{array}{l}\text { Ecologia } \\
\text { Solidarietà } \\
\text { Pace } \\
\text { Omosessuali }\end{array}$ & $\begin{array}{r}84 \\
14 \\
-b \\
2\end{array}$ & $\begin{array}{r}86 \\
10 \\
2 \\
2\end{array}$ & $\begin{array}{r}8 \\
50 \\
20 \\
23\end{array}$ & $\begin{array}{r}77 \\
19 \\
3 \\
0\end{array}$ \\
\hline Totale & 100 & 100 & 100 & 100 \\
\hline
\end{tabular}

- Il dato si riferisce a tre organizzazioni invece che a quattro.

${ }^{b}$ L'informazione non è disponibile.

to Greenpeace: nel 1985, con l'assenso del governo, i servizi segreti francesi fecero esplodere una bomba sul «Rainbow Warrior» in Nuova Zelanda (rimase ucciso un uomo). La sezione francese di Greenpeace ha sofferto molto di questo attentato, perdendo sia membri sia risorse finanziarie.

I risultati inerenti alle risorse finanziarie rispecchiano, grosso modo, quelli relativi alle adesioni. $\mathrm{Li}$ presento unicamente al livello dell'intero campione. Nella tab. 4 viene riportato il livello medio delle risorse a disposizione dell'intero sottosettore delle organizzazioni dei nuovi movimenti sociali per ciascun paese, e dell'infrastruttura organizzativa di ciascun movimento nei quattro paesi considerati insieme ${ }^{19}$. In termini sia assoluti che relativi, le organizzazioni francesi dispongono di un minore numero di membri e di una minore quantità di risorse finanziarie.

19 In questo caso sono state incluse le associazioni di associazioni, in quanto, anche se non hanno membri individuali, hanno un bilancio proprio. 
$\mathrm{TAB}$. 4. Livello medio di adesione (numero di membri) e di risorse finanziarie (in migliaia di dollari) delle organizzazioni di movimento sociale nel 1989 per paese e per movimento

\begin{tabular}{|c|c|c|c|c|c|c|}
\hline & \multicolumn{2}{|c|}{ Adesioni } & \multicolumn{4}{|c|}{ Risorse } \\
\hline & $\begin{array}{c}\text { Valore } \\
\text { assoluto }\end{array}$ & $\begin{array}{l}\text { Per milione } \\
\text { di abitanti }\end{array}$ & $(\mathrm{N})$ & $\begin{array}{c}\text { Valore } \\
\text { assoluto }\end{array}$ & $\begin{array}{l}\text { Per milione } \\
\text { di abitanti }\end{array}$ & (N) \\
\hline \multicolumn{7}{|l|}{ Paese: } \\
\hline $\begin{array}{l}\text { Paesi Bassi } \\
\text { Germania } \\
\text { Francia } \\
\text { Svizzera }\end{array}$ & $\begin{array}{r}59.200 \\
62.700 \\
7.200 \\
16.500\end{array}$ & $\begin{array}{r}3.940 \\
1.020 \\
130 \\
2.550\end{array}$ & $\begin{array}{l}(21) \\
(19) \\
(24) \\
(32)\end{array}$ & $\begin{array}{r}3.940 \\
3.590 \\
580 \\
1.600\end{array}$ & $\begin{array}{r}263 \\
58 \\
10 \\
246\end{array}$ & $\begin{array}{l}(13) \\
(27) \\
(21) \\
(28)\end{array}$ \\
\hline \multicolumn{7}{|l|}{ Movimento: } \\
\hline $\begin{array}{l}\text { Ecologia } \\
\text { Solidarietà } \\
\text { Pace } \\
\text { Omosessuali } \\
\text { Autonomia }\end{array}$ & $\begin{array}{r}91.800 \\
15.500 \\
5.300 \\
5.000 \\
880\end{array}$ & $\begin{array}{r}5.450 \\
1.020 \\
270 \\
170 \\
110\end{array}$ & $\begin{array}{r}(27) \\
(32) \\
(15) \\
(16) \\
(6)\end{array}$ & $\begin{array}{r}4.390 \\
1.850 \\
330 \\
720 \\
170\end{array}$ & $\begin{array}{r}235 \\
144 \\
8 \\
14 \\
24\end{array}$ & $\begin{array}{r}(31) \\
(31) \\
(12) \\
(9) \\
(6)\end{array}$ \\
\hline $\begin{array}{l}\text { Media } \\
\text { complessiva }\end{array}$ & 32.700 & 1.950 & (96) & 2.300 & 132 & (89) \\
\hline
\end{tabular}

In termini assoluti, le organizzazioni olandesi e tedesche sono le più dotate di risorse; in termini relativi, le organizzazioni svizzere sono alla pari con quelle olandesi, mentre quelle tedesche occupano una posizione intermedia. Quanto ai movimenti, quello per l'ecologia è il più dotato; segue il movimento solidaristico. Le organizzazioni del movimento per la pace sono molto più svantaggiate: hanno tanti aderenti quanto il movimento per gli omosessuali, ma dispongono di meno risorse. Il movimento di autonomia, infine, sembra ancora più debole. Sebbene la nostra base di dati per questo movimento sia piuttosto inconsistente, si può affermare che verso la fine degli anni ottanta gli aderenti a questo movimento controculturale e le risorse a sua disposizione fossero esigui. Un dato non riportato nella tab. 4 mostra ancora una volta quanto sia debole il movimento per l'ecologia in Francia, in termini sia di adesioni sia di situazione finanziaria: circa 1 ' $80 \%$ delle risorse del sottosettore dei nuovi movimenti sociali in Francia è controllato da organizzazioni del movimento solidaristico.

I dati permettono un'analisi più approfondita dello sviluppo nel numero di adesioni al movimento per l'ecologia in tre paesi, 
nei quali quest'ultimo domina il sottosettore dei nuovi movimenti sociali. La fig. 4 illustra come le tre o quattro più grandi organizzazioni di questo movimento si sono evolute nel periodo che va dal 1975 al 1989. Complessivamente, tutte e tre le infrastrutture sono cresciute in maniera impressionante nel corso di questo periodo. Se si analizza nei particolari il modello di crescita, si osserva che le tradizionali organizzazioni di orientamento conservazionista - come l'associazione tedesca per la protezione degli uccelli (Dbv) o quella olandese per la tutela della natura (natuurmonumenten) - fondate molto prima dell'avvento dei nuovi movimenti sociali, crescono assai lentamente o rimangono sostanzialmente stabili. Non crescono più di tanto neppure le organizzazioni un po' più vecchie e di orientamento ambientalista - come la Wwf in Svizzera - le quali hanno adottato un approccio pragmatico verso la conservazione e il miglioramento dell'ambiente umano. La forte crescita di queste infrastrutture negli anni ottanta è dovuta soprattutto allo sviluppo assai sostenuto dei movimenti ambientalisti sorti di recente e di orientamento ecologista - ad esempio, l'associazione svizzera per i trasporti (Vcs) e, soprattutto, Greenpeace ${ }^{20}$.

Il modello svizzero si rileva particolarmente degno di nota, in quanto esso si caratterizza per una diversificazione dell'infrastruttura organizzativa del movimento verso un nuovo ambito la politica dei trasporti - alla quale si accompagna un aumento della divisione del lavoro e del coordinamento orizzontale tra le organizzazioni del settore. Di conseguenza, in Svizzera l'appena fondata Vcs si dedica ai problemi del trasporto, la fondazione per l'energia si interessa della politica energetica, la piccola associazione per la tutela dell'ambiente si concentra su problemi come l'inquinamento atmosferico, e la Wwf e la grande federazione per la tutela della natura si occupano soprattutto della conservazione dell'ambiente. A causa dell'atteggiamento competitivo tenuto da Greenpeace nei confronti delle altre organizzazioni in questo ambito, lo sviluppo delle infrastrutture organizzative in Germania e nei Paesi Bassi sembra più fragile rispetto alla Svizzera.

Come ho già sostenuto in precedenza, nelle prime fasi del

${ }^{20}$ La distinzione tra conservazionismo tradizionale, ambientalismo pragmatico ed ecologismo politico è stata concettualizzata da Diani e Lodi (1988) per lo studio del movimento per l'ecologia in Italia, ed è stata applicata da Rucht (1989) ai movimenti per l'ecologia in Germania e in Francia. 

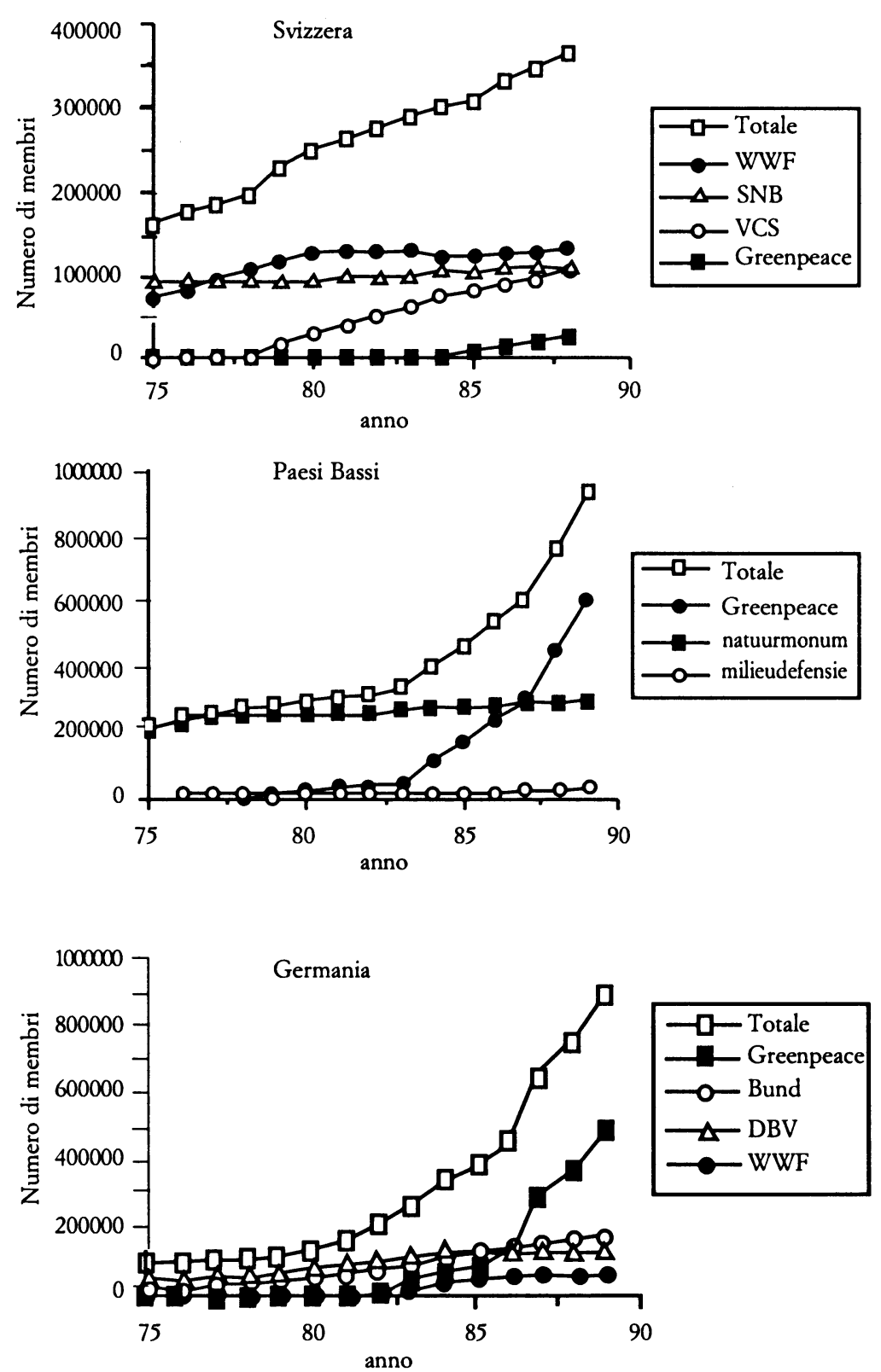

FIG. 4. Evoluzione del numero di aderenti alle maggiori organizzazioni del movimento per l'ecologia in tre paesi (1975-1989) 
processo di mobilitazione di un movimento sociale le sue organizzazioni non possono ancora fare affidamento su un flusso stabile di risorse; devono, invece, ricorrere all'impegno ad boc degli attivisti. Nella misura in cui riescono a sopravvivere alle vicissitudini delle fasi iniziali del processo di mobilitazione e si fanno una buona reputazione, è probabile che le organizzazioni acquisiscano un insieme stabile di aderenti e un flusso continuo di risorse finanziarie. In altri termini, ci si aspetta che la quantità di risorse a disposizione di una determinata organizzazione di movimento sociale sia una funzione non solo del contesto politico e del tipo di infrastruttura in cui opera, ma anche dell'età.

Al fine di controllare questa ipotesi, distinguo nettamente tra organizzazioni fondate prima del 1965 , cioè nate prima della mobilitazione dei nuovi movimenti sociali, e quelle fondate a partire da quella data. Già nel trattare i modelli di crescita del movimento per l'ecologia avevo evidenziato come alcune delle organizzazioni prese in esame siano emerse molto prima dell'avvento dei nuovi movimenti sociali. Il fatto che queste organizzazioni già esistessero quando sono sorti i nuovi movimenti sociali non preclude che esse possano fare parte delle loro infrastrutture organizzative. Anzi, le nuove ondate di mobilitazione hanno rivitalizzato molte organizzazioni tradizionali, le quali hanno adattato i propri obiettivi e i repertori delle azioni ai nuovi movimenti sociali e si sono integrate nelle loro infrastrutture. A prescindere dal contesto politico delle infrastrutture, le organizzazioni tradizionali tendono a disporre di più membri e risorse finanziarie delle organizzazioni fondate dagli stessi nuovi movimenti sociali.

La tab. 5 conferma che le cose stanno proprio così. In media, le organizzazioni tradizionali hanno più membri e più risorse finanziarie delle organizzazioni fondate di recente. C'è, comunque, un'eccezione a questa generalizzazione, un'eccezione così importante da capovolgere la relazione in uno dei quattro paesi: la sezione tedesca di Greenpeace. Se si tiene conto di questa organizzazione, le organizzazioni tedesche fondate di recente dispongono di più risorse rispetto a quelle tradizionali; se si esclude Greenpeace, la Germania si conforma al modello generale. La Germania è un caso particolare in quanto l'ingente mobilitazione prodotta da Greenpeace è di gran lunga superiore a ogni precedente esperienza in quel paese. Questa situazione eccezionale conferma, in un certo senso, quanto si è afferma- 
TAB. 5. Livello medio di risorse per periodo di fondazione e per paese

\begin{tabular}{|c|c|c|c|c|c|c|c|c|}
\hline \multirow[b]{2}{*}{ Paese } & \multicolumn{8}{|c|}{ Data di fondazione } \\
\hline & \multicolumn{2}{|c|}{$\begin{array}{c}\text { Prima del } \\
1965\end{array}$} & \multicolumn{2}{|c|}{$\begin{array}{l}\text { Dopo il } 1965 \\
\quad \text { (con } \\
\text { Greenpeace) }\end{array}$} & \multicolumn{2}{|c|}{$\begin{array}{l}\text { Dopo il } 1965 \\
\quad \text { (senza } \\
\text { Greenpeace) }\end{array}$} & \multicolumn{2}{|c|}{ Totale $^{b}$} \\
\hline \multicolumn{9}{|c|}{$\begin{array}{l}\text { Numero medio di } \\
\text { aderenti } \\
\text { per milione di } \\
\text { abitanti: }\end{array}$} \\
\hline $\begin{array}{l}\text { Paesi Bassi } \\
\text { Germania } \\
\text { Francia } \\
\text { Svizzera }\end{array}$ & $\begin{array}{r}5.710 \\
790 \\
250 \\
6.310\end{array}$ & $\begin{array}{l}(4) \\
(6) \\
(7) \\
(6)\end{array}$ & $\begin{array}{r}3.530 \\
1.140 \\
80 \\
1.680\end{array}$ & $\begin{array}{l}(17) \\
(13) \\
(17) \\
(26)\end{array}$ & $\begin{array}{r}1.080 \\
411 \\
80 \\
1.600\end{array}$ & $\begin{array}{l}(16) \\
(12) \\
(16) \\
(25)\end{array}$ & $\begin{array}{r}3.940 \\
1.020 \\
130 \\
2.550\end{array}$ & $\begin{array}{l}(21) \\
(19) \\
(24) \\
(32)\end{array}$ \\
\hline $\begin{array}{l}\text { Media } \\
\text { complessiva }\end{array}$ & 2.920 & (23) & 1.640 & (73) & 920 & (69) & 2.550 & (96) \\
\hline \multicolumn{9}{|c|}{$\begin{array}{l}\text { Quantità media di risorse } \\
\text { finanziarie (migliaia di } \\
\text { dollari) per milione di } \\
\text { abitanti: }\end{array}$} \\
\hline $\begin{array}{l}\text { Paesi Bassi } \\
\text { Germania } \\
\text { Francia } \\
\text { Svizzera }\end{array}$ & $\begin{array}{r}928 \\
56 \\
16 \\
827\end{array}$ & $\begin{array}{l}(2) \\
(7) \\
(6) \\
(6)\end{array}$ & $\begin{array}{r}142 \\
60 \\
8 \\
89\end{array}$ & $\begin{array}{l}(11) \\
(20) \\
(15) \\
(22)\end{array}$ & $\begin{array}{r}105 \\
31 \\
8 \\
69\end{array}$ & $\begin{array}{l}(12) \\
(19) \\
(14) \\
(21)\end{array}$ & $\begin{array}{r}252 \\
58 \\
10 \\
246\end{array}$ & $\begin{array}{l}(13) \\
(27) \\
(21) \\
(28)\end{array}$ \\
\hline $\begin{array}{l}\text { Media } \\
\text { complessiva }\end{array}$ & 348 & (21) & 70 & (68) & 50 & (64) & 132 & (89) \\
\hline
\end{tabular}

- Il numero di casi su cui sono basati i calcoli è riportato tra parentesi.

${ }^{b}$ I dati riportati in questa colonna appaiono anche nella tab. 4.

to in precedenza in merito al contesto politico tedesco. In passato la partecipazione politica è stata ridotta, e così è risultato basso anche il numero degli iscritti alle organizzazioni tradizionali. Con la crescita della propensione all'impegno, specie tra le generazioni giovani, sono i nuovi arrivati sulla scena politica ad essere avvantaggiati. É significativo, da questo punto di vista, che il livello delle risorse delle organizzazioni tradizionali in Germania sia paragonabile a quello francese, il quale è estremamente basso rispetto alle situazioni olandese e svizzera. Il livello delle risorse delle organizzazioni tedesche recenti, di converso, si avvicina a quello dei due paesi piccoli del campione. Questo risultato conferma l'idea generale della Germania come democrazia sempre più «normale». 


\section{La strutturazione interna}

Si è visto che il contesto politico esercita un effetto notevole sul livello delle risorse a disposizione delle infrastrutture organizzative dei nuovi movimenti sociali. Per quanto riguarda la strutturazione interna delle organizzazioni, invece, esso svolgerà solo un ruolo indiretto. Come accennato in precedenza, ci si aspetta che la strutturazione interna venga alimentata soprattutto dal livello delle risorse e dall'età dell'organizzazione. Più un'organizzazione è vecchia e maggiori sono le risorse di cui dispone, più è probabile che essa si formalizzi, si professionalizzi, differenzi la propria struttura interna. Il contesto politico può influenzare il livello di strutturazione interna in modo indiretto, facilitando la raccolta di risorse o limitandone l'ammontare. In via approssimativa, comunque, si può affermare che la strutturazione interna viene determinata dalle dinamiche organizzative interne.

Dispongo di due indicatori di strutturazione interna, uno per il grado di formalizzazione di un'organizzazione, l'altro per il grado di professionalizzazione. La definizione operativa del grado di formalizzazione consiste di una scala che tiene conto del fatto che l'organizzazione abbia o meno uno status giuridico; che esista o meno un criterio formale di adesione (possibilmente una qualche forma di obbligo finanziario); che siano previste o meno funzioni formali istituzionalizzate; e, se sono previste tali funzioni, che la differenziazione riguardi solo le funzioni fondamentali (presidenza, segreteria e tesoreria) oppure anche alcune speciali. La scala va da un minimo di 1 a un massimo di 5 . Solo una piccola minoranza delle organizzazioni prese in esame posseggono una struttura priva di formalizzazione. Più della metà (il $57 \%$ ) ha una struttura formale secondo almeno quattro dei punti or ora esplicitati. Ciò significa che il grado complessivo di formalizzazione delle organizzazioni del nostro campione è ragguardevole. Il quadro complessivo non assomiglia affatto all'immagine di reti organizzative decentrate, segmentate e informali che i nuovi movimenti sociali solitamente evocano. Va ribadito, comunque, che il campione non è rappresentativo delle organizzazioni dei movimenti in esame, in quanto sono state considerate solo le organizzazioni più importanti nell'ambito di ciascun movimento. Ciononostante, il livello relativamente alto di formalizzazione raggiunto da queste organizzazioni verso la fine degli anni ottanta indica che le reti organiz- 
TAB. 6. Correlazioni bivariate tra alcune caratteristiche della struttura organizzativa $(N=$ 72, escluso Greenpeace)

\begin{tabular}{lcccc}
\hline & a. Età & b. & c. & d. \\
\hline b. Numero di aderenti & 0,42 & & & \\
c. Risorse finanziarie & 0,33 & 0,78 & & \\
d. Formalizzazione & 0,35 & 0,39 & 0,29 & \\
e. Professionalizzazione & 0,32 & 0,68 & 0,64 & 0,42 \\
\hline
\end{tabular}

zative dei nuovi movimenti sociali, quantomeno delle loro componenti centrali, hanno subito un sostanziale cambiamento strutturale.

Per operativizzare il grado di professionalizzazione è stato rilevato il numero di addetti retribuiti. Così si ottiene solo un'idea approssimativa del livello effettivo di professionalizzazione, in quanto non tutti gli addetti retribuiti saranno necessariamente professionalizzati. Le organizzazioni del campione hanno in media 14 addetti retribuiti. Dietro questa media si nascondono grandi differenze: circa un quarto delle organizzazioni non ha alcun addetto retribuito, e un'altra metà ne ha al massimo quattro; d'altra parte, circa il $10 \%$ delle organizzazioni impiega almeno 50 persone.

La formalizzazione e la professionalizzazione sono positivamente correlate tra loro, nonché con il livello di risorse a disposizione e con l'età dell'organizzazione. Queste correlazioni, dal cui calcolo è stato escluso Greenpeace ${ }^{21}$, sono riportate nella tab. 6. A prescindere dal tipo di movimento e dal paese, entrambi i parametri della strutturazione interna sono una funzione positiva dell'età e delle risorse di un'organizzazione, com'era previsto. Ma come si realizzano queste relazioni con l'età e con le risorse? Sappiamo già che le risorse sono una funzione positiva dell'età. Inoltre, si può assumere che il numero degli áderenti cresca direttamente all'aumentare dell'età, e le risorse finanziarie siano una funzione diretta del numero degli aderenti, in quanto i contributi versati dai membri costituiscono la più importante fonte di entrate per un'organizzazione di movimento sociale (vedi oltre). Possiamo pertanto assumere che il grado di formalizzazione sia una funzione diretta dell'età e del numero

${ }^{21}$ Se si include Greenpeace, le correlazioni tra età e livello di risorse si abbassano in modo considerevole (da 0,42 a 0,12 tra età e numero di membri; da 0,33 a 0,21 tra età e risorse finanziarie); le altre correlazioni cambiano poco. 
TAB. 7. Stima di un modello causale mediante Lisrel: coefficienti standardizzati e varianza spiegata $(N=72$, escluso Greenpeace)

\begin{tabular}{lrcccc}
\hline $\begin{array}{l}\text { Variabili } \\
\text { dipendenti }\end{array}$ & a. Età & b. & c. & d. & $\mathbf{R}^{\mathbf{2}}$ \\
\hline b. Numero di aderenti & 0,42 & & & 0,18 \\
c. Risorse finanziarie & - & 0,78 & & & 0,61 \\
d. Formalizzazione & 0,24 & 0,28 & - & & 0,19 \\
e. Professionalizzazione & - & 0,37 & 0,30 & 0,20 & 0,53 \\
Chi quadrato $=0,01,3$ gradi di libertà, $\mathrm{P}=1,0$ & & & \\
\hline
\end{tabular}

di iscritti, ma non dell'ammontare di risorse: è la complessità implicata da un grande numero di membri a spingere verso la formalizzazione.

Analogamente, mano a mano che un'organizzazione invecchia e l'originario impegno ingente dei suoi attivisti si affievolisce, essa richiede un livello minimo di formalizzazione al fine di rendere stabile la propria struttura. A sua volta, la formalizzazione è il primo passo verso la professionalizzazione. Anzi, più è alto il numero dei membri e maggiore è la quantità di risorse a disposizione, maggiore sarà l'esigenza di professionalizzarsi, come maggiori saranno i mezzi richiesti per compiere questo passo.

Questo semplice modello causale è stato controllato mediante un'analisi Lisrel effettuata su un gruppo di 72 organizzazioni per le quali nessuna di queste informazioni era mancante; inoltre, Greenpeace è stato escluso dall'analisi. I risultati, riportati nella tab. 7, si attengono alle aspettative. Tutti i coefficienti sono statisticamente significativi: l'accostamento del modello ai dati è perfetto. I risultati indicano che circa metà della varianza nella professionalizzazione è attribuibile al livello di risorse e al grado di formalizzazione. L'età e il numero di membri, invece, non danno conto altrettanto bene del grado di formalizzazione, per il fatto che quest'ultimo non varia molto nelle organizzazioni del nostro campione. La tabella mostra anche che circa due terzi della varianza nel livello delle risorse finanziarie è dovuta al numero di membri: le maggiori organizzazioni dei nuovi movimenti sociali dipendono non solo dall'impegno ma anche dai contributi finanziari dei propri aderenti. Infine, l'età dà conto di circa un quinto della varianza nei livelli di adesione. 
Le organizzazioni di movimento sociale tedesche e olandesi presentano un grado di professionalizzazione relativamente elevato, in quanto esse hanno, in termini assoluti, un maggiore numero di membri e più risorse finanziarie rispetto alle organizzazioni francesi e svizzere. Il numero medio di addetti varia da 23,5 e 18,2 per la Germania e i Paesi Bassi, da una parte, a 9,2 e 8,6 per la Francia e la Svizzera, dall'altra. Ci si può chiedere per quale motivo le organizzazioni svizzere non siano più professionalizzate di quelle francesi, rispetto alle quali dispongono di molte più risorse. La risposta sta nel particolare contesto politico della Svizzera: la scarsa professionalizzazione delle organizzazioni può essere attribuita al fatto che ancora oggi l'intero sistema politico funziona secondo il principio della milizia - un altro esempio dell'applicazione molto estesa del principio della sussidiarietà in quel paese. Non tutte le organizzazioni svizzere sono non-professionali: il movimento per l'ecologia, ad esempio, è altamente professionalizzato.

In ogni paese le differenze nei gradi di professionalizzazione dei diversi movimenti tendono a rispecchiare i loro livelli di risorse: il movimento per l'ecologia e quello solidaristico tendono, pertanto, ad essere i più professionalizzati; quelli di autonomia e per la pace i meno professionalizzati.

\section{La strutturazione esterna}

È evidente che la strutturazione esterna, diversamente da quella interna, è una funzione diretta del contesto politico. Disponiamo di due indicatori per analizzare questo aspetto dello sviluppo organizzativo: le preferenze partitiche della base; le alleanze strette con soggetti esterni al sottosettore dei nuovi movimenti sociali.

Per quanto riguarda le preferenze partitiche degli aderenti, in praticamente tutti e quattro i paesi i membri delle organizzazioni prese in esame prediligono i partiti della sinistra. Alcune organizzazioni, tuttavia, raccolgono anche simpatizzanti della destra. Non sorprende che, da una parte, le organizzazioni di questo genere siano numerose soprattutto in Svizzera, dove il $43 \%$ delle organizzazioni di movimento sociale raccolgono simpatizzanti della destra, e, dall'altra, in Francia non vi sia neppure un'organizzazione di questo genere. La forte polarizzazione politico-partitica in Francia obbliga tutti gli attori politici a 
TAB. 8. Alleati esterni dei nuovi movimenti sociali: percentuale di organizzazioni che banno stretto certi tipi di alleanze

\begin{tabular}{lcccc}
\hline $\begin{array}{l}\text { Tipo di } \\
\text { alleanza }\end{array}$ & Paesi Bassi & Germania & Francia & Svizzera \\
\hline Socialdemocratici & & & & \\
o socialisti & 25 & 67 & 21 & 24 \\
Comunisti & 6 & 15 & 21 & 14 \\
Nuova sinistra/verdi & 25 & 26 & 53 & 48 \\
Partiti di sinistra & & 67 & 68 & 52 \\
nel complesso & 38 & 37 & 37 & 48 \\
Sindacati & 25 & 33 & 5 & 38 \\
Chiese & 25 & 4 & 0 & 5 \\
Autorità & 44 & $(27)$ & $(19)$ & $(21)$ \\
(N) & $(16)$ & & & \\
\hline
\end{tabular}

schierarsi (Duyvendak 1992), mentre in Svizzera la contrapposizione meno accentuata tra sinistra e destra facilita gli sconfinamenti. La Germania e i Paesi Bassi stanno in una posizione intermedia con, rispettivamente, il $32 \%$ e il $14 \%$ delle organizzazioni che raccolgono aderenti politicamente di destra.

In tre dei quattro paesi la maggior parte delle organizzazioni stringe alleanze con i partiti della sinistra, come mette in rilievo la tab. $8^{22}$. Soltanto due organizzazioni - una olandese e una svizzera - affermano di essere alleate a partiti di destra. In genere, le organizzazioni dei nuovi movimenti sociali tendono ad allearsi con partiti di sinistra; nei diversi paesi, comunque, varia il partito di riferimento. Ad esempio, alla fine degli anni ottanta, la Spd è di gran lunga il partner preferito dalle organizzazioni tedesche; in Francia e in Svizzera, invece, le organizzazioni di movimento sociale preferiscono le alleanze con i verdi o con uno dei partiti minori della nuova sinistra, sorti sulla scia dei movimenti studenteschi degli anni sessanta e da sempre vicini alle cause dei nuovi movimenti sociali. Nei Paesi Bassi i movimenti manifestano delle posizioni ambigue nelle loro scelte di alleanza.

I comunisti sono degli alleati di rilievo per le organizzazioni di movimento sociale, ma in nessun paese, neppure in Francia,

22 I Paesi Bassi costituiscono l'eccezione, ma questa eccezione potrebbe essere più apparente che reale, in quanto potrebbe essere dovuta a dati insufficienti. 
giungono ad avere lo status di partner preferito. È molto forte la tentazione di interpretare questa differenza tra Germania da una parte e Francia e Svizzera dall'altra in base al fatto che la Spd è all'opposizione dall'inizio degli anni ottanta, mentre i socialisti hanno governato la Francia per quasi tutto il decennio e fanno parte della maggioranza di governo in Svizzera sin dal 1959. Nel corso degli anni ottanta, la Spd ha coltivato i rapporti con i nuovi movimenti sociali, ha scelto un candidato per il cancellierato che ha vedute simili a quelle dei membri di questi movimenti e si è alleata con i verdi in molti Länder. I socialisti francesi, invece, hanno per lo più ritirato il proprio appoggio ai nuovi movimenti sociali; Duyvendak (1992) parla addirittura di tradimento.

La tab. 8 riporta alcuni dati relativi ad altri possibili partner per alleanze: i sindacati, le chiese e le autorità. Per quanto riguarda i sindacati, ho già argomentato altrove (Kriesi 1992) che è improbabile che $\mathrm{i}$ sistemi sindacali altamente corporativi, come quello tedesco, facilitino la mobilitazione dei nuovi movimenti sociali. Ho sostenuto, inoltre, che se vi sono sistemi sindacali divisi lungo linee partitiche e dominati da una federazione comunista, come in Francia, vi è la possibilità che un sindacato minoritario possa raccogliere i consensi di alcune parti della nuova classe media, la quale tende ad essere trascurata dalla federazione dominante: la Cfdt francese ne è un esempio. Ho affermato, infine, che i sistemi sindacali come quelli olandese e svizzero, divisi lungo linee confessionali ma comunque rappacificati e integrati nei processi decisionali della politica, offrono il contesto più vantaggioso per i nuovi movimenti sociali. In questi paesi non solo la lotta di classe si è placata, ma la frammentazione dei sistemi sindacali ha favorito la concorrenza intersindacale, dalla quale i nuovi movimenti sociali traggono beneficio.

Con le informazioni a disposizione non si può confermare questa ipotesi, in quanto non ci sono grandi differenze nei livelli di appoggio (ovunque elevati) dei sindacati per i nuovi movimenti sociali. Un esame più approfondito mostra che in ognuno dei quattro paesi l'appoggio dei sindacati si esprime soprattutto verso le organizzazioni dei movimenti per la pace e per la solidarietà, cioè ai due movimenti che sono stati maggiormente politicizzati dalla sinistra: il $38 \%$ delle organizzazioni del movimento per la pace e il $61 \%$ delle organizzazioni del movimento per la solidarietà hanno ricevuto un appoggio dai sindacati. La 
Germania è l'unico paese nel quale i sindacati hanno appoggiato anche alcune organizzazioni del movimento per l'ecologia.

Il movimento pacifista e quello per la solidarietà sono gli unici ad essere appoggiati dalle chiese in tutti i paesi tranne la Francia. Complessivamente, il $25 \%$ delle organizzazioni del movimento pacifista e il $49 \%$ di quelle del movimento solidaristico hanno ricevuto appoggi da questi alleati. È noto, infatti, che le chiese sono impegnate in questi due movimenti. Sorprende, comunque, il fatto che in Francia questo impegno manchi. Viene da chiedersi se questa assenza delle chiese dai due movimenti francesi dipenda o meno in qualche modo dalla forte polarizzazione sinistra-destra che li caratterizza.

I Paesi Bassi si distinguono per l'elevato numero di organizzazioni che hanno stretto alleanze con le autorità. Quasi la metà delle organizzazioni olandesi affermano di essere alleati ad autorità; in Francia, invece, non vi è alcuna alleanza di questo tipo, e ce n'è soltanto una in ciascuno degli altri due paesi. Queste alleanze sono diffuse soprattutto nel movimento olandese per l'ecologia; anzi, tutte le sue organizzazioni hanno stretto alleanze con le autorità. Ciò è una conseguenza diretta del «nuovo realismo» praticato dal movimento per l'ecologia nei Paesi Bassi nel corso degli anni ottanta (Cramer 1989), ma d'altra parte deriva anche dalla strategia di «cooptazione informale» che caratterizza il sistema politico olandese.

La diversificazione dei rapporti di alleanza può rivelarsi di importanza centrale per un'organizzazione di movimento sociale, come si evince dal caso di un'organizzazione svizzera del movimento solidaristico che si era mobilitata a favore dei lavoratori immigrati (Cattacin e Passy 1992). L'organizzazione ricevette consistenti aiuti finanziari da parte del più importante sindacato edile, i cui iscritti provenivano per la grande maggioranza dall'Europa meridionale. L'organizzazione si adoperò attivamente per la difesa degli interessi del sindacato, consentendogli così di mantenere un basso profilo, in relazione alla questione politicamente delicata dello statuto dei lavoratori stagionali. Attenuatasi la salienza politica del problema, il sindacato perse interesse per l'organizzazione e interruppe i suoi aiuti, privandola delle risorse necessarie per sopravvivere.

Il numero di alleati esterni può fungere da indicatore grossolano della diversificazione dei rapporti esterni di un'organiz$z_{\text {zazione }}^{23}$. Secondo questo indicatore, le organizzazioni svizzere

${ }^{23}$ Il questionario usato per la rilevazione si limitava a registrare non più di tre alleati esterni. 
hanno una rete di alleanze esterne più diversificata rispetto alle organizzazioni degli altri paesi. Se si tiene conto del contesto politico, le organizzazioni dei movimenti strumentali - e, tra queste, quelle dei movimenti per la pace e per la solidarietà dispongono di reti più diversificate rispetto ai movimenti sub-e controculturali.

\section{La trasformazione degli obiettivi e dei repertori delle azioni}

La trasformazione degli obiettivi è di difficile operativizzazione se si dispone solo di informazioni di tipo strutturale, come quelle rilevate nell'ambito di questo progetto. È comunque possibile intuire la direzione di questa trasformazione mediante l'analisi delle fonti delle entrate delle organizzazioni. Alle organizzazioni è stato chiesto di indicare l'incidenza sull'insieme delle proprie entrate dei contributi dei membri, dei sussidi pubblici, degli aiuti degli alleati e delle altre fonti. Più è ridotta l'incidenza dei contributi dei membri sul bilancio complessivo, più l'organizzazione è autonoma rispetto ai membri e più è probabile che obiettivi che non siano di immediato interesse per $\mathrm{i}$ membri influenzino la coalizione che controlla l'organizzazione. La distribuzione complessiva delle entrate dell'organizzazione rispetto alle quattro fonti succitate rispecchia il grado di diversificazione della sua base di risorse e il suo grado di autonomia da ambienti specifici. L'incidenza dei sussidi pubblici, in particolare, indica quanto l'organizzazione è integrata nei sistemi esistenti di intermediazione degli interessi, nonché il grado di pragmatismo che informa la scelta degli obiettivi. Si è rilevato anche se l'organizzazione fa o meno ricorso ad incentivi selettivi al fine di aumentare il numero dei membri. La proporzione di organizzazioni che impiegano questo genere di incentivi rivela in modo approssimativo in che misura esse si stanno orientando verso la commercializzazione o l'involuzione.

La trasformazione dei repertori delle azioni delle organizzazioni nei quattro paesi può essere esaminata mediante l'analisi dell'archivio degli eventi di protesta per il periodo che va dal 1976 al 1988. La dinamica dell'entità complessiva delle attività di protesta in questo periodo è stata studiata da Kriesi et al. (1992), che hanno dimostrato che essa è strettamente collegata ai cambiamenti del contesto politico. Ė importante sottolineare che il livello delle attività di protesta dei nuovi movimenti non 
è diminuito; anzi, se si eccettua la Francia, non è mai stato elevato come negli anni ottanta. In questa sede ci occuperemo non dell'entità complessiva della protesta, bensì della trasformazione dei repertori delle azioni: in che misura essi sono diventati più estremi oppure più convenzionali?

Consideriamo, innanzitutto, la misura in cui le organizzazioni di movimento sociale fanno ricorso ad incentivi selettivi per attirare nuovi membri. La seconda parte della tab. 9 mostra che non vi sono differenze sistematiche tra i vari paesi. Pare che gli incentivi selettivi siano particolarmente diffusi nel movimento degli omosessuali, a prescindere dal paese. Ciò si conforma all'ipotesi che i movimenti subculturali tendano ad orientarsi verso l'involuzione o la commercializzazione.

Alcune differenze tra i paesi si riscontrano, invece, per quanto attiene alle fonti di entrata. Le organizzazioni svizzere dipendono dai contributi dei membri in misura maggiore rispetto alle organizzazioni degli altri paesi. È necessario sottolineare, comunque, che per la Svizzera si dispone di informazioni sulle fonti di entrata limitatamente alle sole organizzazioni del movimento per la solidarietà. Tuttavia, la base di risorse di que-

TAB. 9. Fonti di entrata delle organizzazioni di movimento sociale (incidenza percentuale sulle entrate totali) e ricorso a incentivi selettivi (percentuale di organizzazioni che vi ricorrono)

\begin{tabular}{|c|c|c|c|c|c|c|c|}
\hline & \multicolumn{4}{|c|}{ Fonti di entrata } & \multicolumn{3}{|c|}{$\%$ ricorso a } \\
\hline & Aderenti & Sussidi & Alleati & Altre & $(\mathrm{N})$ & selettivi & $(\mathrm{N})$ \\
\hline \multicolumn{8}{|l|}{ Paese: } \\
\hline $\begin{array}{l}\text { Paesi Bassi } \\
\text { Germania } \\
\text { Francia } \\
\text { Svizzera }\end{array}$ & $\begin{array}{l}55 \\
60 \\
61 \\
81\end{array}$ & $\begin{array}{r}22 \\
16 \\
9 \\
5\end{array}$ & $\begin{array}{r}7 \\
14 \\
8 \\
1\end{array}$ & $\begin{array}{r}16 \\
9 \\
22 \\
13\end{array}$ & $\begin{array}{l}(16) \\
(23) \\
(25) \\
(12)\end{array}$ & $\begin{array}{l}46 \\
39 \\
44 \\
50\end{array}$ & $\begin{array}{l}(24) \\
(28) \\
(25) \\
(32)\end{array}$ \\
\hline \multicolumn{8}{|l|}{ Movimento: } \\
\hline $\begin{array}{l}\text { Ecologia } \\
\text { Solidarietà } \\
\text { Pace } \\
\text { Omosessuali } \\
\text { Autonomia }\end{array}$ & $\begin{array}{l}55 \\
56 \\
68 \\
60 \\
94\end{array}$ & $\begin{array}{r}23 \\
12 \\
2 \\
14 \\
5\end{array}$ & $\begin{array}{r}5 \\
16 \\
21 \\
4 \\
0\end{array}$ & $\begin{array}{r}17 \\
16 \\
9 \\
22 \\
0\end{array}$ & $\begin{array}{r}(22) \\
(18) \\
(9) \\
(13) \\
(2)\end{array}$ & $\begin{array}{l}49 \\
28 \\
31 \\
81 \\
67\end{array}$ & $\begin{array}{r}(35) \\
(36) \\
(16) \\
(16) \\
(6)\end{array}$ \\
\hline $\begin{array}{l}\text { Media } \\
\text { complessiva }\end{array}$ & 63 & 13 & 8 & 16 & (78) & 45 & (109) \\
\hline
\end{tabular}


ste ultime si rivela particolarmente poco diversificata, sia che venga paragonata alle basi di risorse di tutte le organizzazioni degli altri paesi sia che si prendano in considerazione solo le organizzazioni del movimento per la solidarietà. Dunque, il sottosettore svizzero che dispone della rete di alleanze più diversificata è allo stesso tempo quello che più si affida ai propri mezzi. Ciò rispecchia perfettamente la specificità del contesto politico svizzero: quest'ultimo consente un ampio grado di consultazione e concertazione politica $\mathrm{e}$, in base al principio di sussidiarietà, attribuisce molta importanza all'auto-sufficienza e all'autoorganizzazione (Kriesi 1980).

Nel contesto olandese, al contrario, oltre alla promozione della concertazione, vi è la tendenza ad erogare aiuti pubblici alle organizzazioni: i movimenti per l'ecologia, per la solidarietà e degli omosessuali beneficiano tutti di considerevoli sussidi. A titolo di esempio, si possono confrontare le fonti di entrata del Comitato per il Nicaragua nei Paesi Bassi e del Comitato per l'America centrale in Svizzera: il primo è finanziato per il $60 \%$ da autorità pubbliche, il secondo dipende per il $90 \%$ dai contributi dei propri membri e per il rimanente $10 \%$ da «altre» fonti. Il Comitato per il Nicaragua in Francia raccoglie il 50\% dei finanziamenti dai propri membri e il $50 \%$ da «altre» fonti; in genere, le «altre fonti» sono più importanti in Francia, controbilanciando così la mancanza di appoggi governativi e il basso numero di aderenti.

I sussidi pubblici sono rilevanti soprattutto per i movimenti per l'ecologia e degli omosessuali, i quali vengono assistiti non solo nei Paesi Bassi ma anche negli altri paesi. Il $45 \%$ del bilancio della $\mathrm{Bbu}$, l'associazione che riunisce i gruppi civici per la tutela ambientale in Germania, è coperto da sussidi pubblici, i quali incidono per circa un terzo sui bilanci degli Amis de la Terre e della Ffspn (l'associazione che riunisce i gruppi per la tutela ambientale in Francia). In Svizzera il movimento per l'ecologia ancora non riceve aiuti pubblici; ne riceve invece il movimento degli omosessuali a causa del fenomeno dell'Aids. Ovunque i governi collaborano con le organizzazioni dei movimenti subculturali, i quali fungono da intermediari rispetto a gruppi a rischio ai quali le autorità non avrebbero altrimenti accesso. La crisi dell'Aids è un esempio delle «emergenze che improvvisamente si impongono», le quali, prescindendo dal contesto politico, ristrutturano i rapporti esterni di un'infrastruttura organizzativa e ne trasformano drasticamente gli obiettivi. 
Sono le organizzazioni dei movimenti pacifista (in Francia) e solidaristico (nei Paesi Bassi e in Germania) ad avere più probabilità di ricevere finanziamenti da alleati. In Francia, le maggiori organizzazioni del movimento per la pace sono alleate al partito comunista (Mouvement de la Paix, Appel des Cent) o alla nuova, o «seconda», sinistra (Codene, il comitato per il disarmo nucleare in Europa). Circa il $40-50 \%$ delle loro entrate sono dovute a questi alleati. Il fatto che questi ultimi pongano, com'era prevedibile, delle condizioni sottolinea quanto detto in precedenza circa la politicizzazione del movimento pacifista francese.

Quanto alla trasformazione dei repertori delle azioni, prendiamo in esame le tendenze relative alla convenzionalizzazione $o$, viceversa, alla radicalizzazione di quattro dei cinque movimenti. Non verrà preso in esame il movimento degli omosessuali, in quanto ha prodotto pochissimi eventi di protesta. Per questa analisi verranno impiegati i dati relativi agli eventi di protesta rilevati mediante un'analisi del contenuto della stampa quotidiana ${ }^{24}$. In questa sede distinguerò tra eventi di protesta convenzionali e non convenzionali. Gli eventi convenzionali comprendono le azioni legali (ad esempio, citare qualcuno in tribunale), le azioni politiche convenzionali (ad esempio, partecipare a una procedura di consultazione), azioni convenzionali dirette ai mezzi di informazione (come le conferenze stampa e le dichiarazioni pubbliche) e gli scioperi, nella misura in cui questi eventi sono stati riportati nei quotidiani consultati. Sono state considerate eventi non convenzionali tutte le azioni pubbliche di natura dimostrativa, conflittuale o violenta riportate nei quotidiani esaminati.

La fig. 5 presenta l'incidenza annua degli eventi non convenzionali messi in atto dai tre movimenti strumentali. I dati raffigurati sono delle medie mobili che tengono conto dei trienni precedenti e successivi ad ogni anno; lo smussamento delle curve che ne risulta mette in maggiore evidenza le tendenze sottostanti. Per il movimento di autonomia i dati si riferiscono a un sottoinsieme degli eventi non convenzionali, precisamente a quelli violenti, i quali sono molto più diffusi in questo movimento che non negli altri di tipo strumentale.

24 Seguendo l'impostazione di altre ricerche (Tilly et al. 1975; Kriesi et al. 1981; McAdam 1983; Tarrow 1989), sono state raccolte informazioni sistematiche sugli eventi di protesta mediante l'esame dell'edizione del lunedi di un quotidiano importante per tutto il periodo $1976-1988$. 

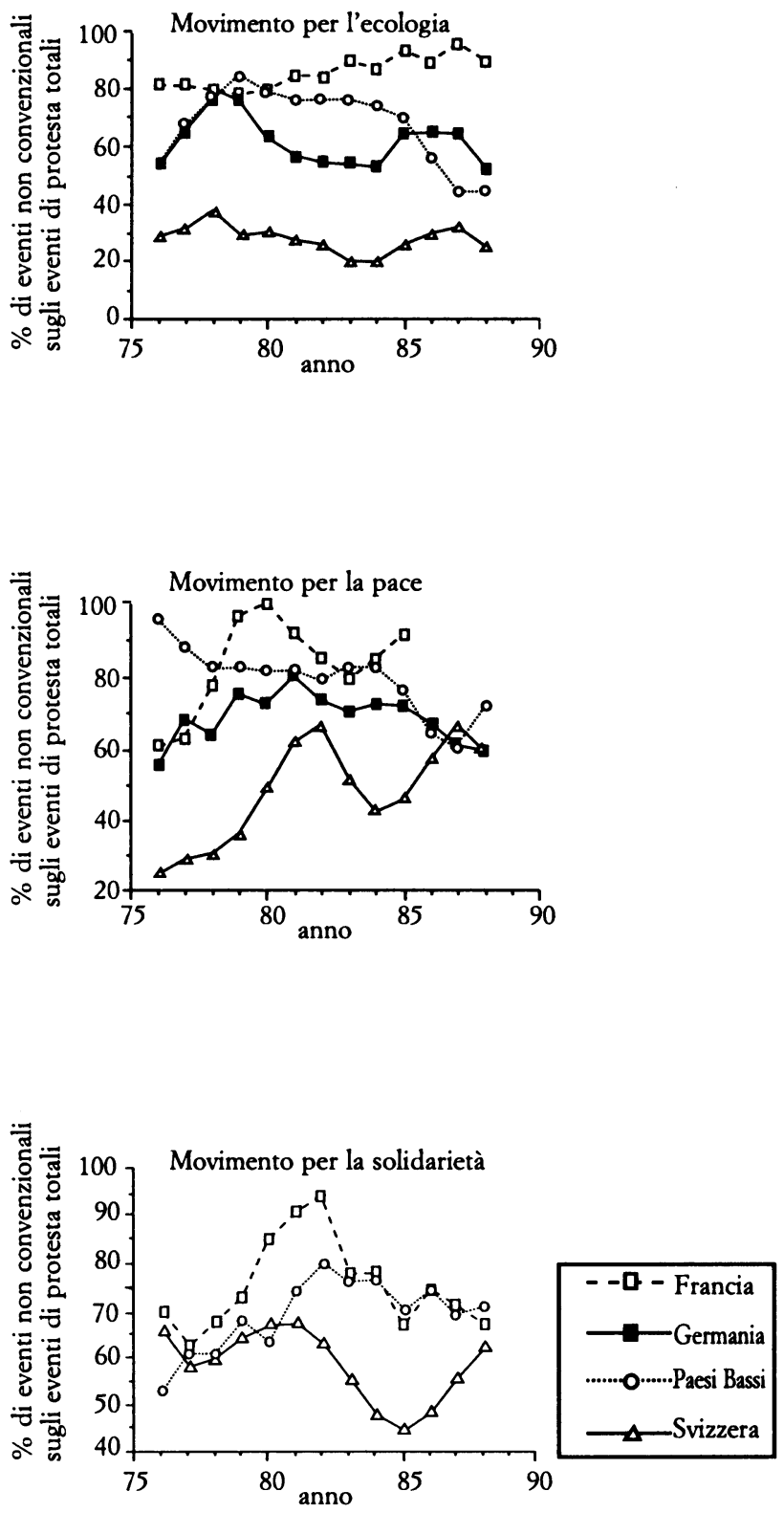

FIG. 5. Evoluzione dei repertori delle azioni di quattro nuovi movimenti sociali (19761988) 
Il primo risultato degno di nota è che ogni paese presenta delle differenze attinenti al repertorio delle azioni nell'ambito di ciascuno dei quattro movimenti. I movimenti francesi hanno i repertori meno convenzionali, ovvero i più violenti; quelli svizzeri sono i più convenzionali; quelli tedeschi e olandesi si situano in una posizione intermedia. Queste differenze corrispondono alle aspettative che si sarebbero potute formulare a partire dalle diverse strutture di opportunità politica nei quattro paesi: in Francia, il contesto di «esclusione selettiva» incoraggia le proteste virulente; in Svizzera, il contesto di «integrazione procedurale totale» favorisce le sfide moderate e convenzionali; i due contesti intermedi producono livelli intermedi di radicalizzazione.

Oltre a queste diversità sistematiche nei modelli generali, la fig. 5 mette in risalto altre importanti differenze inerenti alle dinamiche temporali relative ai movimenti presi singolarmente e nel loro insieme; e anche queste differenze sono collegate ai contesti politici. Nel caso del movimento per l'ecologia, si osserva una progressiva radicalizzazione in Francia, mentre nei Paesi Bassi e in Germania vi è una forte tendenza alla convenzionalizzazione. Alla fine degli anni settanta, in seguito alla loro radicalizzazione nel corso del dibattito sull'energia nucleare, i movimenti tedesco e olandese presentavano dei repertori di azioni tanto estremi quanto quello dei colleghi francesi. Tuttavia, a partire dai primi anni ottanta, $i$ repertori tedeschi e olandesi sono diventati più convenzionali e si sono avvicinati a quello dell'assai convenzionale movimento svizzero; il movimento francese, nel frattempo, si è ulteriormente radicalizzato. Queste diversità rispecchiano le modifiche intervenute nella configurazione del potere in Francia e in Germania: quando i socialisti andarono al potere in Francia nel 1981, il movimento per l'ecologia perse il suo più potente alleato; nel 1982, quando la Spd andò all'opposizione in Germania, il movimento trovò un nuovo alleato. Nel caso olandese la convenzionalizzazione è una conseguenza del «nuovo realismo» che caratterizza il movimento per l'ecologia a partire dalla sconfitta del suo ramo anti-nucleare all'inizio degli anni ottanta.

Anche per quanto riguarda il movimento per la pace si osservano una progressiva radicalizzazione in Francia e tendenze verso la convenzionalizzazione in Germania e nei Paesi Bassi ${ }^{25}$.

${ }^{25}$ Nella fig. 5, la curva che rappresenta il movimento pacifista francese termina prima delle altre perché a partire dal 1986 non sono stati più individuati eventi di protesta messi in atto da questo movimento. 
Il movimento svizzero ha attraversato due periodi di radicalizzazione, i quali l'hanno avvicinato ai movimenti olandese e tedesco. Il primo periodo di radicalizzazione era determinato dalla situazione internazionale: così come avvenne nei paesi della Nato, anche gli svizzeri furono mobilitati dalla campagna contro i missili cruise. Il secondo periodo, invece, era un fatto interno legato alla campagna per l'abolizione dell'esercito svizzero. La moderazione negli altri due paesi è la conseguenza della fine della lunga campagna contro i missili cruise; nei Paesi Bassi, la moderazione si manifesta a partire dalla fine del 1985 , quando il governo decise di ospitare i missili sul territorio nazionale.

Per quanto riguarda il movimento per la solidarietà, in Svizzera si riscontrano ancora una volta due distinte ondate di radicalizzazione. La prima ondata segue una tendenza presente anche in Francia e nei Paesi Bassi, evidenziando ancora una volta l'effetto della situazione internazionale ${ }^{26}$. Ma, mentre gli altri due movimenti continuano a radicalizzarsi, quello svizzero diventa molto convenzionale fino alla metà degli anni ottanta per poi radicalizzarsi di nuovo durante le campagne a favore dei rifugiati politici, intraprese in risposta alla posizione intransigente del governo su questo problema. Il movimento francese, invece, ricorre ad azioni sempre meno estreme mano a mano che aumenta l'appoggio da parte dei socialisti al governo.

Infine, si differenziano anche le tendenze relative al movimento di autonomia. In Francia esso è sempre stato molto piccolo ed estremamente radicale, ma tende ad ammorbidirsi verso la fine degli anni ottanta. Anche in Svizzera e nei Paesi Bassi il movimento si deradicalizza in seguito a un periodo di radicalizzazione. In Germania, invece, si manifesta la tendenza opposta. Sebbene il movimento di autonomia sia in genere molto radicale, esistono dei limiti oltre ai quali il processo di radicalizzazione non può andare. In un certo senso, questo processo porta alla propria sconfitta: il movimento provoca azioni sempre più repressive da parte delle autorità - azioni che peraltro tendono ad essere efficaci - e, inoltre, tende a spegnersi in quanto gli attivisti, molto semplicemente, si esauriscono. Nel caso del movi-

${ }^{26}$ La curva relativa al movimento tedesco per la solidarietà è stata esclusa dal grafico per non complicarne la lettura. Il movimento tedesco esprime una deradicalizzazione fino alla metà degli anni ottanta, per poi radicalizzarsi nella seconda metà degli anni ottanta; così la sua curva si sarebbe incrociata due volte con quelle francese e olandese. 
mento di autonomia, la dinamica dell'interazione con le autorità si estrinseca soprattutto a livello locale; pertanto non è possibile interpretarne le tendenze in relazione ai contesti politici nazionali.

Naturalmente, gli eventi di protesta imputabili a un movimento non sono necessariamente messi in atto dalla sua infrastruttura organizzativa. Alcuni eventi possono essere organizzati dagli alleati, altri ancora effettuati senza alcun appoggio organizzativo. Tuttavia; è probabile che l'evoluzione generale del repertorio delle azioni di un movimento porti i segni dello sviluppo della sua base organizzativa e abbia degli effetti su di esso. Per sostanziare questo asserto, effettuerò un'analisi del repertorio delle azioni delle organizzazioni del movimento per l'ecologia $^{27}$. Ai fini di questa analisi, la distinzione tra organizzazioni moderate e radicali viene operativizzata distinguendo tra le grandi organizzazioni professionali da una parte e i piccoli gruppi informali di azione civica dall'altra ${ }^{28}$.

Come si è già visto, se si eccettua la Francia il repertorio delle azioni del movimento per l'ecologia era diventato piuttosto convenzionale verso la fine degli anni ottanta. Questa deradicalizzazione può derivare $\mathrm{da}$ uno spostamento di iniziativa dalle organizzazioni radicali a quelle moderate, oppure da una progressiva moderazione di gruppi che in precedenza erano radicali, oppure da entrambi i processi. Le informazioni della fig. 6 - la quale riporta il numero complessivo di eventi di protesta e il numero di eventi non convenzionali messi in atto dalle organizzazioni professionali e dai gruppi di azione civica in ognuno dei tre paesi in cui il movimento si è deradicalizzato - permettono di separare questi due effetti. La fig. 6 non si limita a riportare le tendenze (come faceva la fig. 5), ma indica anche il livello di mobilitazione delle diverse organizzazioni.

In ciascuno dei tre paesi la mobilitazione dei gruppi di azione civica è notevolmente diminuita verso la fine degli anni ottanta. Questa smobilitazione ha contribuito alla deradicalizzazione del movimento in Germania e nei Paesi Bassi, ma non in

${ }^{27}$ Il numero degli eventi di protesta imputabili ad organizzazioni del movimento per l'ecologia varia nei quattro paesi: in Francia, esse hanno partecipato solo al $15 \%$ di questi eventi, mentre negli altri paesi la partecipazione sale al $42.45 \%$ (Germania e Svizzera) o al 55\% (Paesi Bassi).

${ }^{28}$ I quotidiani spesso riportano i nomi delle organizzazioni che sono responsabili per avere promosso un evento di protesta. Questa informazione è stata usata per distinguere tra le organizzazioni professionali e i gruppi informali di azione civica. 

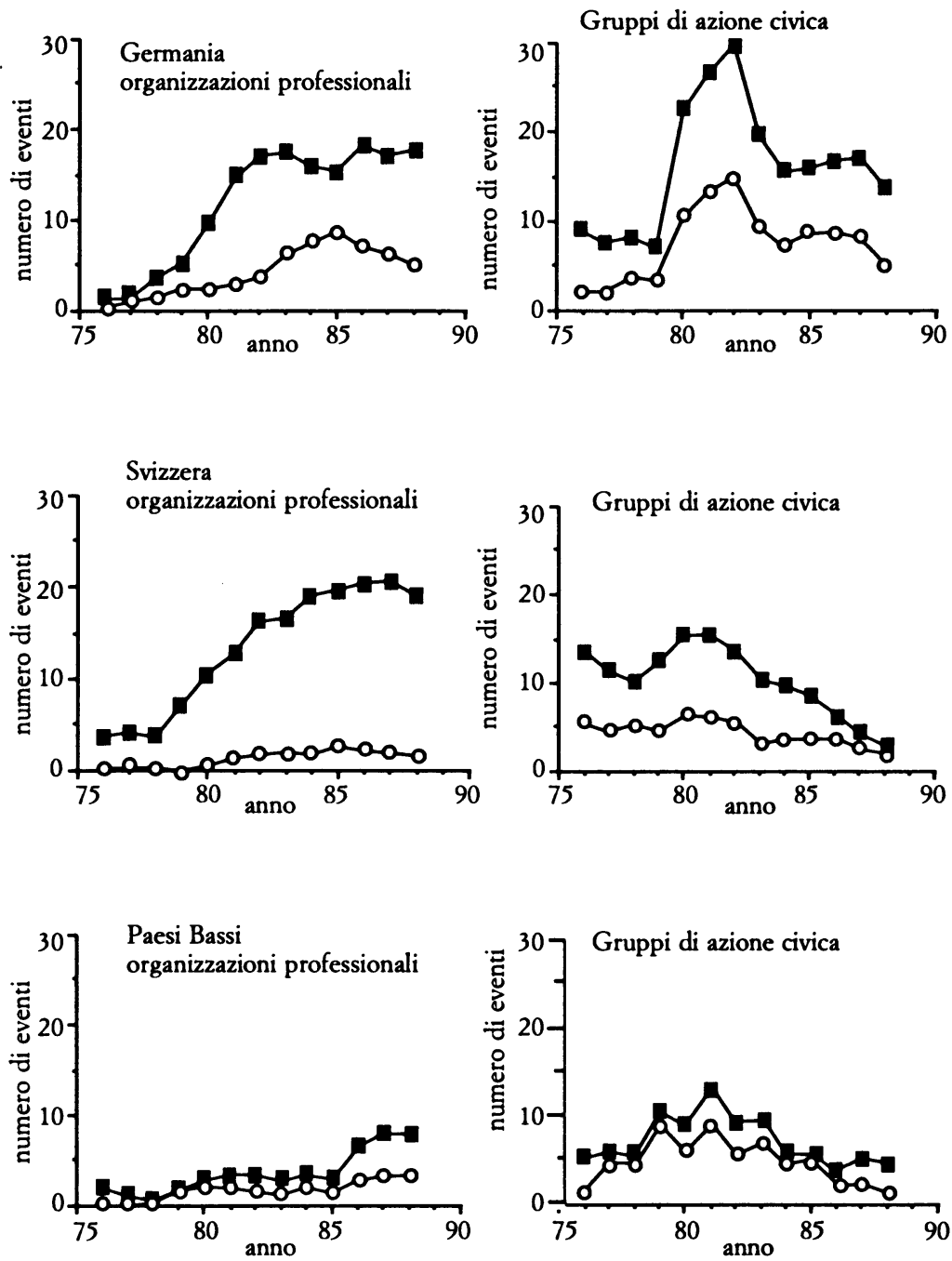

$$
\begin{aligned}
& - \text { Eventi totali } \\
& \text {-o- Eventi non } \\
& \text { convenzionali }
\end{aligned}
$$

FIG. 6. Evoluzione dei repertori delle azioni delle organizzazioni del movimento per l'ecologia in tre paesi (1976-1988): organizzazioni professionali e gruppi di azione civica 
Svizzera. In quest'ultimo paese i gruppi di azione civica sono sempre stati piuttosto convenzionali, e la smobilitazione ha provocato soprattutto una diminuzione delle loro attività convenzionali. In Germania e in Svizzera, la smobilitazione dei gruppi di azione civica si è accompagnata a un aumento delle attività di protesta da parte delle organizzazioni professionali. La mobilitazione delle associazioni professionali ha prodotto azioni quasi esclusivamente convenzionali in Svizzera, un po' più radicali in Germania. Nei Paesi Bassi, infine, l'iniziativa non è passata alle organizzazioni professionali: sembra che la smobilitazione dei gruppi di azione civica abbia comportato una smobilitazione dell'intero movimento.

La differenza tra la Germania e la Svizzera da una parte e i Paesi Bassi dall'altra può essere interpretata alla luce di un risultato commentato in precedenza: in quest'ultimo paese il movimento per l'ecologia si è integrato nei sistemi di intermediazione degli interessi, viene riconosciuto dalle autorità e ne riceve sussidi in una misura assai superiore che non in Svizzera. Per dirla altrimenti, le organizzazioni professionali nei Paesi Bassi non hanno più bisogno di mobilitare la gente per far sì che il loro punto di vista venga rappresentato nel processo politico e per far sì che qualcosa venga fatto. Anche in Svizzera e in Germania il movimento ha maggiori possibilità di accedere al sistema, come testimonia la convenzionalizzazione del suo repertorio delle azioni. Tuttavia, persino le organizzazioni professionali continuano a ricorrere alla mobilitazione della protesta (in forme soprattutto convenzionali) al fine di ottenere qualcosa. Come il movimento per l'ecologia nel suo complesso è di gran lunga il più istituzionalizzato tra $\mathrm{i}$ cinque movimenti presi in esame, così le organizzazioni del movimento per l'ecologia nei Paesi Bassi sono le più vicine al diventare veri e propri gruppi di interesse ${ }^{29}$.

29 I partiti verdi, pur essendo un frutto della mobilitazione dei nuovi movimenti sociali, non sono un prodotto della trasformazione di organizzazioni preesistenti. Ovunque $\mathrm{i}$ partiti verdi sono stati istituiti ex novo. $\mathrm{Li}$ considererei un alleato esterno dei nuovi movimenti sociali piuttosto che una componente delle loro infrastrutture organizzative. Mediante le loro alleanze con l'infrastruttura organizzativa del movimento per l'ecologia, i partiti verdi hanno, naturalmente, favorito la sua istituzionalizzazione. 


\section{Conclusioni}

In questo saggio ho analizzato i fattori interni ed esterni che influenzano lo sviluppo organizzativo dei nuovi movimenti sociali, evidenziando soprattutto gli effetti del contesto politico senza tuttavia limitare lo sguardo ai soli fattori politici. Se l'ambiente politico generale e la configurazione del potere nel sistema politico sono centrali per lo sviluppo dell'infrastruttura organizzativa dei nuovi movimenti sociali, essi non rappresentano gli unici fattori importanti. In primo luogo, questa analisi ha confermato che la strutturazione interna dipende soprattutto dalla dinamica organizzativa interna. Inoltre, essa mette in evidenza alcune differenze importanti nello sviluppo organizzativo dei diversi movimenti che prescindono dal contesto politico. Ad esempio, il movimento per l'ecologia tende ad essere il più dotato di risorse.

Sono state riscontrate delle disparità tra i movimenti per quanto riguarda i livelli di appoggio esterno: in genere, i movimenti per la pace e per la solidarietà ricevono un forte sostegno dalla sinistra, compresi i sindacati, e dalle chiese; il movimento degli omosessuali, invece, riceve aiuti pubblici in tutti i paesi a causa del diffondersi dell'Aids. Ancora, com'era previsto, i diversi tipi di movimento subiscono diverse forme di trasformazione: i nostri indicatori, per quanto siano inadeguati, forniscono indizi favorevoli all'ipotesi che i movimenti subculturali tendano alla commercializzazione o all'involuzione, mentre quelli istituzionali tendano ad istituzionalizzarsi. Tra i movimenti istituzionali, quelli per la solidarietà e per la pace hanno maggiori difficoltà a stabilizzarsi rispetto al movimento per l'ecologia, in quanto la loro particolare struttura dei problemi li rende vulnerabili alle variazioni congiunturali del ciclo dell'attenzione pubblica e alla strumentalizzazione politica. Il movimento per l'ecologia è quello che si è più istituzionalizzato, avvicinandosi alla forma organizzativa del gruppo di interesse.

Esistono delle eccezioni a queste generalizzazioni, eccezioni dovute agli specifici contesti politici dei paesi presi in esame. In Francia, ad esempio, la debolezza e il basso livello di istituzionalizzazione del movimento per l'ecologia, nonché la relativa forza del movimento per la solidarietà, derivano dalle strategie attuate dal governo socialista. Il grado inspiegabilmente basso di professionalizzazione delle organizzazioni svizzere appare in- 
vece ragionevole se lo si interpreta in relazione alla centralità del sistema della milizia nella sfera politica di quel paese. In Germania, analogamente, la forte influenza dell'età di un'organizzazione sul suo livello di risorse può essere interpretata alla luce del divario che tradizionalmente separa stato e società in quel paese.

Il contesto politico è importante non solo per spiegare i casi devianti rispetto ai modelli previsti per i movimenti, ma anche perché è stata dimostrata la sua grande influenza sui livelli delle risorse, sulla strutturazione esterna e sulla trasformazione degli obiettivi e dei repertori delle azioni. Le organizzazioni di movimento sociale francesi, ad esempio, tendono ad essere deboli, estremiste e fortemente strumentalizzate dai partiti. Le organizzazioni nei due paesi più piccoli, invece, possono fare affidamento su un numero relativamente elevato di membri e su molte risorse, sono relativamente ben integrate nei sistemi di intermediazione degli interessi e tendono a ricorrere ad azioni moderate. Le organizzazioni olandesi godono del più alto livello di risorse in termini assoluti, ma intrattengono anche $\mathrm{i}$ rapporti più articolati con le autorità. Le organizzazioni svizzere si distinguono da quelle olandesi in quanto hanno diversificato in modo esteso le proprie alleanze e i propri modelli di reclutamento, ma non ricevono quasi alcun aiuto dalle autorità. Le organizzazioni tedesche, infine, si situano in una posizione intermedia sulla maggior parte degli aspetti dello sviluppo organizzativo.

In generale, queste risultanze confermano l'importanza del contesto politico nella mobilitazione dei nuovi movimenti sociali. Esse confermano altresì le specifiche ipotesi che sono state formulate in relazione alle differenze di sviluppo organizzativo tra $\mathrm{i}$ movimenti che non possono essere attribuite al contesto politico.

[traduzione di Giancarlo Gasperoni]

Riferimenti bibliografici

Almond, G.A. e S. Verba (1963), The Civic Culture. Political Attitudes and Democracy in Five Nations, Princeton, Princeton University Press.

Bader, V. (1992), Kollektives Handeln, Opladen, Leske und Budrich. 
Cattacin, S. e F. Passy (1992), Der Niedergang von Bewergungsorganisationen. Zur Analyse von organisatorischen Laufbabnen, relazione presentata al convegno su «New Social Movements, the Third Sector and the Welfare State», Bonn, 26-29 giugno.

Cramer, J. (1989), De groene golf. Geschiednenis en toekomst van de milieubeweging, Utrecht, Uitgeverij Jan van Arkel.

Curtis, J.E., E.G. Grabb e D.E. Baer (1992), Voluntary Association Membership in Fifteen Countries: A Comparative Analysis, in «American Sociological Review», LVII, n. 2, pp. 139-152.

Diani, M. e G. Lodi (1988), Three in One: Currents in the Milan Ecology Movement, in Klandermans et al. (1988), pp. 103-124.

Duyvendak, J.W. (1992), The Power of Politics: New Social Movements in an Old-fashioned Polity, Università di Amsterdam, tesi di dottorato.

Gamson, W.A. (1975), The Strategy of Social Protest, Homewood, Dorsey.

Garner Ash, R. e M.N. Zald (1987), The Political Economy of Social Movement Sectors, in Zald e McCarthy (1987), pp. 293-318.

Gerlach, L.P. e V.H. Hine (1970), People, Power and Change: Movements of Social Transformation, Indianapolis, Bobbs-Merrill.

Goldstone, J.A. (1980), The Weakness of Organization: A New Look at Gamson's «The Strategy of Social Protest», in «American Journal of Sociology», LXXXV, pp. 1017-1042.

Hannan, M.T. e J. Freeman (1984), Structural Inertia and Organizational Change, in «American Sociological Review», XLIX, n. 2, pp. 149-164.

Hardin, R. (1982), Collective Action, Baltimore, Johns Hopkins University Press.

Jenkins, J.C. e C.M. Eckert (1986), Channeling Black Insurgency, in «American Sociological Review», LI, n. 6, pp. 812-829.

Kanter, R.M. (1972), Commitment and Community, Cambridge, Mass., Harvard University Press.

Klandermans, B. (1988), The Formation and Mobilization of Consensus, in Klandermans et al. (1988), pp. 173-196.

- (a cura di) (1989) International Social Movement Research, Greenwich, Jai-Press, vol. 2.

Klandermans, B., H. Kriesi e S. Tarrow (a cura di) (1988), International Social Movement Research, Greenwich, Jai-Press, vol. 1.

Kleinert, H (1991), Die Grünen 1990/91. Vom Wabldebakel zum Neuanfang, in «Aus Politik und Zeitgeschichte», B44, pp. 27-37.

Koopmans, R. (1990a), Bridging the Gap: The Missing Link between Political Opportunity Structure amd Movement Action, relazione presentata al congresso dell'Associazione mondiale di sociologia, Madrid, luglio.

- (1990b), Patterns of Unruliness: The Interactive Dynamics of Pro- 
test Waves, manoscritto non pubblicato, Università di Amsterdam, Pdis.

- (1992), Democracy from Below. New Social Movements and the Political System in Germany, Università di Amsterdam, tesi di dottorato.

Kriesi, H. (1980), Entscheidungsstrukturen und Entscheidungsprozesse in der Schweizer Politik, Frankfurt, Campus.

- (1989), New Social Movements and the New Class in the Netherlands, in «American Journal of Sociology», XCIV, n. 5, pp. 10781116.

- (1990), Federalism and Pillarization: The Netherlands and Switzerland Compared, in «Acta politica», XXV, n. 4, pp. 433-450.

- (1991), The Political Opportunity Structure of New Social Movements: Its Impact on Their Mobilization, FS III 91-103, Berlin, Wissenschaftszentrum.

Kriesi, H., R. Koopmans, J.W. Duyvendak e M.G. Giugni (1992), New Social Movements and Political Opportunities in Western Europe, in «European Journal of Political Research», XXII, pp. 219244.

Kriesi, H., R. Levy, G. Ganguillet e H. Zwicky (a cura di) (1981), Politische Aktivierung in der Schweiz: 1945-78, Grüsch, Rüegger.

McAdam, D. (1982), Political Process and the Development of Black Insurgency: 1930-1970, Chicago, University of Chicago Press.

- (1983), Tactical Innovation and the Pace of Insurgency, in «American Sociological Review», XLVIII, pp. 725-753.

McAdam, D., J.D. McCarthy e M.N. Zald (1988), Social Movements, in N.J. Smelser (a cura di), Handbook of Sociology, London, Sage, pp. 695-738.

McCarthy, J.D. e M.N. Zald (1977), Resource Mobilization and Social Movements: A Partial Theory, in «American Journal of Sociology», LXXXII, n. 6, pp. 1212-1241.

- (1987), Appendix. The Trend of Social Movements in America: Professionalization and Resource Mobilization, in Zald e McCarthy (1987), pp. 337-392.

Melich, A. (a cura di) (1992), Les valeurs des Suisses, Berne, Lang.

Meyer, J.W. e B. Rowan (1977), Instituzionalized Organizations: Formal Structure as Myth and Ceremony, in «American Journal of Sociology», LXXXIII, n. 2, pp. 364-385.

Morris, A.D. (1984), The Origins of the Civil Rights Movement, New York, Free Press.

Oberschall, A. (1978), The Decline of the 1960s Social Movements, in L. Kriesberg (a cura di), Research in Social Movements: Conflict and Change, vol. III, Greenwich, Jai-Press, pp. 257-290.

Olson, M. (1965), The Logic of Collective Action, Cambridge, Mass., Harvard University Press; trad. it., La logica dell'azione collettiva, Feltrinelli, Milano, 1983. 
Piven, F.F. e R.A. Cloward (1977), Poor People's Movements, New York, Vintage.

Raschke, J. (1991), Krise der Brünen. Bilanz und Neubeginn, Marburg, Schüren.

Rosanvallon, P. (1988), La question syndicale, Paris, Calman-Levy.

Roth, R. (1991), Herausforderung demokratischer Institutionen durch neue Formen politischer Mobilisierung. Zur Situation in der Bundesrepublik, in «Schweiz. Jahrbuch für Politische Wissenschaft», XXXI, pp. 209-234.

Rucht, D. (1989), Environmental Movement Organizations in West Germany and France: Structure and Interorganizational Relations, in Klandermans (1989), pp. 61-94.

Rudzio, W. (1987), Das politische System der Bundesrepublik Deutschland, Leverkusen, Utb, $2^{\mathrm{a}}$ ed.

Schmitter, P.C. e W. Streeck (1981), The Organization of Business Interests. A Research Design to Study the Associative Action of Business in the Advanced Industrial Societies of Western Europe, Berlin, Wissenschaftszentrum.

Sontheimer, K. (1989), Grundzüge des politischen Systems der Bundesrepublik Deutschland, München, Piper, $12^{\mathrm{a}}$ ed.

Staggenborg, S. (1988), The Consequences of Professionalization and Formalization in the Pro-Choice Movement, in «American Sociological Review», LIII, n. 4, pp. 585-605.

Tarrow, S. (1989), Democracy and Disorder. Protest and Politics in Italy: 1965-1975, Oxford, Clarendon Press; trad. it. Democrazia e disordine, Bari, Laterza, 1990.

Taylor, V. (1989), Social Movement Continuity: The Women's Movement in Abeyance, in «American Sociological Review», LIV, n. 5, pp. 761-775.

Tilly, C. (1978), From Mobilization to Revolution, Reading, AddisonWesley.

Tilly, C., L. Tilly e R. Tilly (1975), The Rebellious Century: 1830-1930, Cambridge, Mass., Harvard University Press.

Van Praag, P. Jr. (1992), De smalle marges van een brede bewging. Vredesprotest in Nederland, in J.W. Duyvendak et al. (a cura di), Tussen verbeelding en macht. 25 jaar nieuwe sociale bewegingen in Nederland, Amsterdam, Sua, pp. 99-120.

Zald, M.N. e R. Ash (1966), Social Movement Organizations: Growth, Decay and Change, in «Social Forces», XLIV, n. 3, pp. 327-340.

Zald, M.N. e J.D. McCarthy (a cura di) (1987), Social Movements in an Organizational Society, New Brunswick, Transaction.

Zucker, L.G. (1987), Institutional Theories of Organization, in «Annual Review of Sociology», XIII, pp. 443-464. 